\title{
Integral equation approach to tropical ocean dynamics: Part II-Rossby wave scattering from the equatorial Atlantic western boundary
}

\author{
by Paulo R. Holvorcem ${ }^{1,2}$ and Marcio L. Vianna'
}

\begin{abstract}
We develop a linear theory for the scattering of equatorial waves of a fixed frequency $\omega$ by islands and continental margins of arbitrary geometry by use of the boundary integral equation (BIE) method of Vianna and Holvorcem (Part I of this work). All the solutions of the equatorial $\beta$-plane dispersion relations at frequency $\omega$ are treated explicitly through the extensive use of exact Green's functions, so that the approach is more general and more rigorous than previous attempts to solve equatorial scattering problems, many of which employ the low-frequency and long-wave approximations. The numerical solution of the BIE is obtained through application of the boundary element method. A numerical study of the scattering of Rossby waves with periods between 50 and 90 days from the equatorial Atlantic western boundary is presented. Some of the resulting interference patterns exhibit a sharp amplitude maximum, whose center lies between 3-9N, 35-47W. The position, width and intensity of this maximum all depend on wave period. We find evidences that this maximum arises from the superposition of zonally damped equatorial modes (evanescent waves) excited at the western boundary. The largest pressure amplitudes along the boundary are found in the southern hemisphere between the Equator and $5 \mathrm{~S}$. The phase propagation along the boundary is generally northwestward, except at a few positions where the phase is stationary. We discuss similarities and differences between the calculated responses and observations of intraseasonal oscillations in the tropical Atlantic Ocean.
\end{abstract}

\section{Introduction}

The generation of equatorial waves from non-uniform large scale wind systems and their subsequent scattering by ocean boundaries is believed to play a central role in the adjustment of the upper ocean thermal structure, which interacts back with the atmosphere through the generation of sea surface temperature anomalies (see, e.g., Enfield, 1989). Recent results obtained from analysis of Geosat sea level anomaly fields for the Pacific warm pool area revealed the importance of low frequency

1. Divisāo de Ciências da Terra, Instituto Nacional de Pesquisas Espaciais, Av. dos Astronautas 1758, São José dos Campos, SP, 12201, Brazil.

2. Present address: Instituto de Matemática, Estatística e Ciência da Computacāo, Universidade Estadual de Campinas, C.P. 6065, Campinas, SP, 13081, Brazil. 
Rossby wave reflection from the Pacific western boundary in relation to El Niño (Delcroix et al., 1989; Picaut et al., 1989).

On the other hand, large amplitude intraseasonal oscillations, interpreted as being possibly due to westward propagating Rossby waves, have been found in current meter mooring records just off the western boundary of the equatorial Atlantic at 52W (Johns et al., 1990). These oscillations, however, are not confined to the immediate vicinity of the boundary, but have a considerably larger zonal extent, as evidenced by mean sea-level and sea surface temperature data from the Gulf of Guinea reported by Picaut and Verstraete (1976) and by the current meter data at 28W reported by Richardson and Reverdin (1987) and Weisberg et al. (1987). Furthermore, inverted echo-sounder (IES) data from SEQUAL (1983-84) taken at $28 \mathrm{~W}$ indicate the presence of strong intraseasonal oscillations in the 50-100 day period range (Garzoli, 1987). All these measurements were made either along or north of the Equator. In a series of papers, Weisberg and Tang $(1985,1987,1990)$ have shown that the seasonal wind-forced equatorial Atlantic thermocline response far from boundaries can be accounted for by a linear superposition of equatorial long waves. This was done by calculating time-dependent interference patterns, with adjusted positions for the meridional model boundaries.

Similar results for 50-day waves were found in the western equatorial Indian Ocean by Luyten and Roemmich (1982); these seem to be well described by Kindle and Thompson's (1989) 11/2-layer model forced by realistic winds. Instabilities of the surface zonal and western boundary currents are invoked as possible generators of the observed oscillations with periods of 50 and 26 days.

In a recent study, Moore and McCreary (1990) forced a model Indian Ocean with periodic winds at this frequency range, in order to examine the effects of wave reflection at the western boundary on the basin-wide response. In their study, short equatorial waves generated at the western boundary are not filtrated. The chosen grid resolution $(50 \mathrm{~km})$ was adjusted to include the effects of the shortest wave modes present at the 60-day period in the second baroclinic mode of the tropical Indian Ocean. Moore and McCreary implemented slip boundary conditions by use of a staggered grid and a straight boundary making an angle of $45^{\circ}$ to the Equator. Since the considered frequencies are intermediate, this kind of study cannot rely on the long wave or low frequency approximations, which impose "averaged" boundary conditions at the western boundary (Cane and Gent, 1984; McCalpin, 1987). The interference patterns which result from the excitation of 60-day equatorial waves in the Indian Ocean present an intense maximum south of the Equator in the vicinity of the western boundary.

The effects of islands on the propagation of equatorial waves is also an important issue that should be considered. This problem has been studied numerically by Yoon (1981) using a finite difference method and by Rowlands (1982) using an integral equation approach which somewhat resembles the spirit of the present series of 
papers. However, Rowlands did consider only the idealized case of a meridionally oriented island of negligible zonal extension. The effects of one such "thin island" on the propagation of low-frequency equatorial waves have also been studied by Cane and du Penhoat (1982). Recently, in connection with the reflection and transmission of equatorial waves by the irregular western Pacific boundary, this theory has been generalized to include several thin islands (Clarke, 1991a) and western boundary gaps (du Penhoat and Cane, 1989). The high sensitivity of experiments with such very idealized geometries points out to the need for numerical calculations where the detailed boundary geometry is taken into account (du Penhoat and Cane, 1989).

In the present paper, the boundary integral equation formulation of the linearized equatorial $\beta$-plane shallow water equations introduced in Part I of this work (Vianna and Holvorcem, 1992; henceforth Part I) is adapted to describe the general scattering problem for time-harmonic equatorial waves. Through the extensive use of exact Green's functions, our method can deal rigorously with the entire range of frequencies of oceanographic interest. No equatorial mode is neglected a priori in constructing the Green's functions, since the previous studies of Part I have demonstrated that there are regions in the fields of the Green's functions where the short wavelength modes and the zonally damped modes (evanescent waves) make a crucial contribution. The scattering problem is formulated for an arbitrary boundary geometry, and there is no difficulty in applying exactly the slip boundary conditions. The boundary integral equations of the theory are efficiently solved for the boundary pressure distribution by the boundary element method (Brebbia et al., 1984). Once this pressure distribution is known, the method enables the calculation of the dynamical variables at any point within the ocean, even near the boundaries. This can be very important in connection with the study of time series obtained from current meter moorings and IES's, since if well-defined oscillations are found in a narrow band of the power spectrum, one can determine what spatial distribution of intensities one should expect on a basin-wide scale.

A previous attempt to calculate the interior ocean response from sea level anomalies at the ocean boundaries was presented by Gill (1983), who showed that the whole dynamics of equatorial motions during El Niño could possibly be retrieved from time series of coastal sea level anomaly, once the wind stress field was known. In that work, sea surface temperature and zonal surface current anomalies in the central Pacific during the 1972 El Niño were reconstructed from eastern boundary sea level anomalies. The converse process, i.e., to extract the signature of equatorial waves from coastal sea-level records, is also possible, and has been accomplished using data from the eastern Pacific boundary (Clarke, 1991b).

The plan of the paper is as follows. In Section 2, we review the results of Part I which are needed in the present study. Section 3 presents the derivation of boundary integral equations describing the scattering of Kelvin and Rossby waves with westward group velocity, and in Section 4 we discuss numerical methods for their 
solution. In Section 5 we present the results of a numerical study on the scattering of Rossby waves with periods between 50 and 90 days by a model western Atlantic boundary of realistic horizontal geometry (a vertical wall at the $200 \mathrm{~m}$ isobath around a flat bottom ocean with imposed vertical eigenvalues appropriate to the Atlantic); we compare our results with some recent observations of intraseasonal oscillations in the equatorial Atlantic (Richardson and Reverdin, 1987; Garzoli, 1987; Johns et al., 1990). Finally, in Section 6 we discuss the main conclusions of the paper.

\section{Review of previous results}

In Part I of this work the linearized shallow water equations on the $\beta$-plane,

$$
\begin{gathered}
\rho_{o}\left(\partial_{t} \mathbf{u}+\beta y \hat{\mathbf{z}} \times \mathbf{u}\right)=-\nabla p-\left(\rho_{o} A / c^{2}\right) \mathbf{u}+\mathbf{F}, \\
\partial_{t} p+\left(A / c^{2}\right) p+\rho_{o} c^{2} \nabla \cdot \mathbf{u}=0,
\end{gathered}
$$

which describe the dynamics of a single baroclinic mode with vertical eigenvalue $c$, were reformulated as a boundary integral equation (BIE). In (2.1) and (2.2), $\rho_{o}$ is a scale density of water, $p, \mathbf{u}$ and $\mathbf{F}$ denote the projections of pressure, velocity and wind-stress onto the given baroclinic mode, and $A$ is a constant which enters in the parameterization of vertical diffusion of heat and momentum through the relation (McCreary, 1981)

$$
\kappa=\nu=A / N^{2}(z),
$$

where $\kappa$ and $\nu$ are the diffusion coefficients of heat and momentum and $N$ is the Brunt-Väisälä frequency.

The analysis in Part I was performed in the frequency domain, all dependent variables being complex functions of the position vector $\mathbf{r}=(x, y)$ and the frequency $\omega$ (we often omit the argument $\omega$ for brevity). In this paper, we consider only waves of a fixed frequency, so that any dependent variable $f$ can be obtained from the relation

$$
f(\mathbf{r}, t)=\operatorname{Re}\left\{e^{i \omega} f(\mathbf{r}, \omega)\right\} .
$$

After Fourier transformation, (2.1) and (2.2) can be reduced to a single differential equation for the horizontal structure of the pressure perturbation,

$$
\mathscr{L} p=S
$$

where

$$
\begin{gathered}
\mathscr{L}=\mathscr{L}\left(y_{c}\right)=\nabla \cdot\left[\left(y^{2}-y_{c}^{2}\right)^{-1}\left(y \hat{\mathbf{z}} \times-i y_{c}\right) \nabla\right]+i y_{c} / 4, \\
\quad S=\nabla \cdot\left[\left(y^{2}-y_{c}^{2}\right)^{-1}\left(y \hat{\mathbf{z}} \times-i y_{c}\right) \mathbf{F}^{\prime}\right], \\
y_{c}=(\omega-i \Delta \omega) / \omega_{o}, \quad \Delta \omega=A / c^{2}, \quad \omega_{o}=\beta R_{o}, \\
R_{o}=(c / 2 \beta)^{1 / 2}, \quad \mathbf{F}^{\prime}=R_{o} \mathbf{F} .
\end{gathered}
$$

As in Part I, the spatial coordinates $x$ and $y$ are scaled by the equatorial deformation 
radius $R_{o}$. The velocity field can be written in terms of the pressure perturbation as

$$
\mathbf{u}=2\left(\rho_{o} c\right)^{-1}\left(y^{2}-y_{c}^{2}\right)^{-1}\left(i y_{c}-y \hat{\mathbf{z}} \times\right)\left(\mathbf{F}^{\prime}-\nabla p\right) .
$$

The pressure perturbation field within any fluid region $B$ satisfies the integral identity

$$
\begin{aligned}
\varphi\left(\mathbf{r}^{\prime}\right)= & \mathrm{P} . \mathrm{V} . \oint_{\Gamma} p(\mathbf{r}) \mathbf{K}\left(\mathbf{r}^{\prime} ; \mathbf{r}\right) \cdot \hat{\mathbf{n}}(s) d s-1 / 2 \rho_{o} c \oint_{\Gamma} q(s) G\left(\mathbf{r}^{\prime} ; \mathbf{r}\right) d s \\
& -\iint_{B} \mathbf{F}^{\prime}(\mathbf{r}) \cdot \mathbf{K}\left(\mathbf{r}^{\prime} ; \mathbf{r}\right) d x d y
\end{aligned}
$$

where $\Gamma=\partial B$,

$$
\epsilon=\left\{\begin{array}{cc}
1, & \text { for } \mathbf{r}^{\prime} \text { inside } B \\
1 / 2, & \text { for } \mathbf{r}^{\prime} \text { on } \Gamma \\
0, & \text { for } \mathbf{r}^{\prime} \text { outside } B,
\end{array}\right.
$$

$s$ is a coordinate measuring arclength along $\Gamma, \mathbf{r}=\mathbf{r}(s)$ varies along $\Gamma, \hat{\mathbf{n}}(s)$ is the outer normal vector to $\Gamma, q=\mathbf{u} \cdot \hat{\mathbf{n}}$ denotes the normal outflow at the boundary and P.V. denotes the principal value. The kernels $G\left(\mathbf{r}^{\prime} ; \mathbf{r}\right)$ and $\mathbf{K}\left(\mathbf{r}^{\prime} ; \mathbf{r}\right)$ which appear in (2.10) are the basic influence functions associated with the propagation of pressure perturbations on the equatorial $\beta$-plane. When $\mathbf{r}^{\prime}$ is an interior point of $B,(2.10)$ is an integral representation for the interior pressure field in terms of its boundary values; when $\mathbf{r}^{\prime}$ is on $\Gamma,(2.10)$ is a BIE for the boundary pressure distribution $p(s)=p(\mathbf{r}(s))$.

The meridional mode expansions of $G$ and $\mathbf{K}$ are

$$
\begin{aligned}
G\left(\mathbf{r}^{\prime} ; \mathbf{r}\right)= & 2^{-3 / 2}(1-\sigma)\left\{\exp \left[-i \alpha_{-2}\left(x^{\prime}-x\right)\right] \psi_{o}\left(y^{\prime} / \sqrt{2}\right) \psi_{o}(y / \sqrt{2})\right. \\
& \left.+\exp \left[-i \alpha_{-1}\left(x^{\prime}-x\right)\right] \psi_{1}\left(y^{\prime} / \sqrt{2}\right) \psi_{1}(y / \sqrt{2})\right\} \\
& -2^{-1 / 2} \gamma \sum_{m=0}^{\infty} \frac{\exp \left[-i\left(\sigma \lambda_{m}-\gamma\right)\left(x^{\prime}-x\right)\right]}{\lambda_{m}\left(\sigma \lambda_{m}-\lambda_{-1}\right)\left(\sigma \lambda_{m}-\lambda_{-2}\right)} \phi_{m}^{\sigma}\left(y^{\prime}\right) \phi_{m}^{\alpha}(y)
\end{aligned}
$$

$$
\begin{aligned}
\mathbf{K}\left(\mathbf{r}^{\prime} ; \mathbf{r}\right)= & 2^{-5 / 2}(\sigma-1)\left\{\exp \left[-i \alpha_{-2}\left(x^{\prime}-x\right)\right] \psi_{o}\left(y^{\prime} / \sqrt{2}\right) \psi_{o}(y / \sqrt{2}) \hat{\mathbf{x}}\right. \\
& \left.+\exp \left[-i \alpha_{-1}\left(x^{\prime}-x\right)\right] \psi_{1}\left(y^{\prime} / \sqrt{2}\right)\left[\psi_{1}(y / \sqrt{2}) \hat{\mathbf{x}}-4 i \gamma \psi_{o}(y / \sqrt{2}) \hat{\mathbf{y}}\right]\right\} \\
& +2^{-1 / 2} \gamma \sum_{m=0}^{\infty} \frac{\exp \left[-i\left(\sigma \lambda_{m}-\gamma\right)\left(x^{\prime}-x\right)\right]}{\lambda_{m}\left(\sigma \lambda_{m}-\lambda_{-1}\right)\left(\sigma \lambda_{m}-\lambda_{-2}\right)} \phi_{m}^{\sigma}\left(y^{\prime}\right)\left[\zeta_{m}^{\sigma}(y) \hat{\mathbf{x}}+i \theta_{m}^{\sigma}(y) \hat{\mathbf{y}}\right],
\end{aligned}
$$

where

$$
\begin{aligned}
\sigma & =\operatorname{sign}\left(x-x^{\prime}\right), \quad \alpha_{-2}=y_{c} / 2, \quad \alpha_{-1}=y_{c} / 2-y_{c}^{-1}, \\
\gamma & =\left(2 y_{c}\right)^{-1}, \quad \lambda_{m}=i(m+3 / 2-Q)^{1 / 2}, \\
Q & =(1 / 4)\left(y_{c}^{2}+y_{c}^{-2}\right), \quad \lambda_{-1}=\alpha_{-1}+\gamma, \\
\lambda_{-2} & =\alpha_{-2}+\gamma,
\end{aligned}
$$


$\psi_{m}$ denotes the normalized Hermite function of order $m$, and

$$
\begin{aligned}
\phi_{m}^{\mathrm{u}}(y) & =(m+1)^{1 / 2} y \psi_{m+1}(y / \sqrt{2})+2 \alpha_{-2}\left(\sigma \lambda_{m}-\lambda_{-1}\right) \psi_{m}(y / \sqrt{2}), \\
\zeta_{m}^{\mathrm{o}}(y) & =2^{-1}(m+1)^{1 / 2} y \psi_{m+1}(y / \sqrt{2})-\left[m+1+\alpha_{-2}\left(\sigma \lambda_{m}-\lambda_{-1}\right)\right] \psi_{m}(y / \sqrt{2}), \\
\theta_{m}^{\mathrm{s}}(y) & =(m+1)^{1 / 2}\left(\sigma \lambda_{m}-\lambda_{-2}\right) \psi_{m+1}(y / \sqrt{2}) .
\end{aligned}
$$

(These expressions may be easily derived from those given in Part I for $\phi_{m}^{\alpha}, \zeta_{m}^{\sigma}$ and $\theta_{m}^{\alpha}$ by use of the recurrence relations of the Hermite functions.) The parameters $\alpha_{-2}$ and $\alpha_{-1}$ are the wavenumbers of the Kelvin and Yanai waves, respectively; for small friction, and low (high) enough frequency, the wavenumbers of the propagating Rossby (inertia-gravity) modes are given by $\pm \lambda_{m}-\gamma, m=0,1, \ldots, \llbracket \operatorname{Re} Q-3 / 2 \rrbracket$ (the brackets $\llbracket \rrbracket$ denote the integer part); the wavenumbers of the equatorial modes which are zonally damped at frequency $\omega$ are given by $\pm \lambda_{m}-\gamma, m>\llbracket \operatorname{Re} Q-3 / 2 \rrbracket$.

The functions in (2.15) satisfy the following identity, which will be needed in the next section:

$$
\begin{aligned}
& \left(y^{2}-y_{c}^{2}\right)^{-1}\left(i y_{c} \pm y \hat{\mathbf{z}} \times\right) \nabla\left\{\exp \left[ \pm i\left(\sigma \lambda_{m}-\gamma\right) x\right] \phi_{m}^{\sigma}(y)\right\}= \\
& \pm \exp \left[ \pm i\left(\sigma \lambda_{m}-\gamma\right) x\right]\left[\zeta_{m}^{o}(y) \hat{\mathbf{x}} \pm i \theta_{m}^{\sigma}(y) \hat{\mathbf{y}}\right]
\end{aligned}
$$

We refer to Part I for a full discussion on the numerical computation of the influence functions and on their near-field (i.e., as $\mathbf{r} \rightarrow \mathbf{r}^{\prime}$ ) singular behavior.

\section{BIE Formulation of scattering problems}

In this section, we specialize (2.10) to describe the scattering of equatorial waves by islands and continental margins of arbitrary geometry. Consider first an archipelago, formed by the islands (closed contours) $C_{1}, C_{2}, \ldots$ Suppose that a free equatorial wave of frequency $\omega$ incides on the archipelago along the zonal direction. The resulting pressure and velocity fields can be written as

$$
p=p^{(l)}+p^{(S)}, \quad \mathbf{u}=\mathbf{u}^{(l)}+\mathbf{u}^{(S)},
$$

where $p^{(l)}, \mathbf{u}^{(l)}$ are the fields of the incident wave and $p^{(S)}, \mathbf{u}^{(S)}$ are the fields scattered by the archipelago.

In order to apply the integral identity (2.10) to this situation, we take $B$ as the fluid region exterior to the islands and bounded by the meridians $x=x_{W}$ and $x=x_{E}$, which lie respectively to the west and to the east of the archipelago. Since there is no wind forcing in this problem, the identity (2.10) becomes

$$
\begin{aligned}
\varphi\left(\mathbf{r}^{\prime}\right)= & \sum_{j} \operatorname{P.V} . \oint_{C_{j}}\left[p(\mathbf{r}) \mathbf{K}\left(\mathbf{r}^{\prime} ; \mathbf{r}\right)-1 / 2 \rho_{o} c G\left(\mathbf{r}^{\prime} ; \mathbf{r}\right) \mathbf{u}(\mathbf{r})\right] \cdot \hat{\mathbf{n}}(s) d s \\
& +\left[Q^{(l)}(x)+Q^{(s)}(x)\right]_{\substack{k=x_{E} \\
r=x_{W^{\prime}}}}
\end{aligned}
$$


where

$$
\begin{aligned}
& Q^{(l)}(x)=\hat{\mathbf{x}} \cdot \int_{-\infty}^{\infty}\left[p^{(l)}(x, y) \mathbf{K}\left(\mathbf{r}^{\prime} ; x, y\right)-1 / 2 \rho_{o} c G\left(\mathbf{r}^{\prime} ; x, y\right) \mathbf{u}^{(l)}(x, y)\right] d y, \\
& Q^{(s)}(x)=\hat{\mathbf{x}} \cdot \int_{-\infty}^{\infty}\left[p^{(s)}(x, y) \mathbf{K}\left(\mathbf{r}^{\prime} ; x, y\right)-1 / 2 \rho_{o} c G\left(\mathbf{r}^{\prime} ; x, y\right) \mathbf{u}^{(s)}(x, y)\right] d y .
\end{aligned}
$$

A more compact form for (3.2) can be obtained by letting $x_{W} \rightarrow-\infty$ and $x_{E} \rightarrow+\infty$. In the presence of an arbitrarily small amount of friction $(A \neq 0)$, it is known that a free equatorial wave decays exponentially in the direction of its group velocity. In this case, the influence functions $G\left(\mathbf{r}^{\prime} ; \mathbf{r}\right)$ and $\mathbf{K}\left(\mathbf{r}^{\prime} ; \mathbf{r}\right)$ will decay exponentially as $|x| \rightarrow \infty$, since each of these functions represents a superposition of free equatorial waves emitted from $x=x^{\prime}$ (see Part I). The scattered fields consist of waves emitted from the archipelago, and by the same reason they must vanish exponentially as $|x| \rightarrow \infty$. In conclusion, we have that $Q^{(S)}(x) \rightarrow 0$ as $|x| \rightarrow \infty$, and that $Q^{(I)}(x) \rightarrow 0$ for $c_{g} x \rightarrow+\infty$, where $c_{g}$ is the group velocity of the incident wave. However, we shall see shortly that $Q^{(I)}(x)$ is constant for $c_{g} x$ negative and $\left|c_{g} x\right|$ sufficiently large. This happens because the incident fields grow exponentially as $c_{\xi} x \rightarrow-\infty$, while the influence functions multiplying them in (3.3) decay exponentially, so that the integral can approach a constant value.

Here we shall treat two relevant cases: (a) an incident long Rossby wave and (b) an incident Kelvin wave. In the first case, the incident pressure field is

$$
p^{(l)}(\mathbf{r})=p_{o} \exp \left[-i\left(\lambda_{n}-\gamma\right) x\right] \phi_{n}^{+}(y),
$$

where $n=0,1,2, \ldots$ and $p_{o}$ is an arbitrary constant. The velocity field associated with (3.5) is, by (2.9) and (2.16),

$$
\mathbf{u}^{(l)}(\mathbf{r})=\left(2 p_{o} / \rho_{o} c\right) \exp \left[-i\left(\lambda_{n}-\gamma\right) x\right]\left[\zeta_{n}^{+}(y) \hat{\mathbf{x}}-i \theta_{n}^{+}(y) \hat{\mathbf{y}}\right] .
$$

In case (b), the incident pressure field is

$$
p^{(I)}(\mathbf{r})=p_{o} \exp \left(-i \alpha_{-2} x\right) \psi_{o}(y / \sqrt{2})
$$

and the velocity is

$$
\mathbf{u}^{(I)}(\mathbf{r})=\left(p_{o} / \rho_{o} c\right) \exp \left(-i \alpha_{-2} x\right) \psi_{o}(y / \sqrt{2}) \hat{\mathbf{x}}
$$

To compute $Q^{(I)}(x)$, we will need the following identities, which are valid for all $k, m \geq 0$ :

$$
\begin{gathered}
\int_{-\infty}^{\infty} \psi_{k}(y / \sqrt{2}) \psi_{m}(y / \sqrt{2}) d y=2^{1 / 2} \delta_{k m}, \\
\int_{-\infty}^{\infty} y \psi_{k}(y / \sqrt{2}) \psi_{m}(y / \sqrt{2}) d y=2^{1 / 2}\left(k^{1 / 2} \delta_{k-1, m}+m^{1 / 2} \delta_{k, m-1}\right),
\end{gathered}
$$




$$
\begin{aligned}
\int_{-\infty}^{\infty} y^{2} \psi_{k}(y / \sqrt{2}) \psi_{m}(y / \sqrt{2}) d y= & 2^{1 / 2}\left\{(2 k+1) \delta_{k m}\right. \\
& \left.+[k(k-1)]^{1 / 2} \delta_{k-2, m}+[m(m-1)]^{1 / 2} \delta_{k, m-2}\right] .
\end{aligned}
$$

The second and third identities can be deduced from the basic orthogonality relation (3.9) by use of the recurrence formula

$$
y \psi_{m}(y / \sqrt{2})=(m+1)^{1 / 2} \psi_{m+1}(y / \sqrt{2})+m^{1 / 2} \psi_{m-1}(y / \sqrt{2})
$$

CASE (a). Since long Rossby waves have $c_{g}<0$, the previous discussion implies that $Q^{(I)}\left(x_{W}\right) \rightarrow 0$ when $x_{W} \rightarrow-\infty$, so that we need only to evaluate $Q^{(I)}\left(x_{E}\right)$ for $x_{E}$ large enough. By (2.12) and (2.13), we have for $x_{E}>x^{\prime}$ :

$$
\begin{aligned}
P^{(l)}\left(x_{E}\right)= & 2^{-1 / 2} \gamma p_{o} \exp \left[-i\left(\lambda_{n}-\gamma\right) x_{E}\right] \int_{-\infty}^{\infty}\left\{\sum_{m=0}^{\infty} \frac{\exp \left[-i\left(\lambda_{m}-\gamma\right)\left(x^{\prime}-x_{E}\right)\right]}{\lambda_{m}\left(\lambda_{m}-\lambda_{-1}\right)\left(\lambda_{m}-\lambda_{-2}\right)}\right. \\
& \left.\times \phi_{m}^{+}\left(y^{\prime}\right)\left[\zeta_{m}^{+}(y) \phi_{n}^{+}(y)+\zeta_{n}^{+}(y) \phi_{m}^{+}(y)\right]\right\} d y .
\end{aligned}
$$

To simplify (3.13), we will use the following identity, which is a direct consequence of (2.15) and (3.9)-(3.11):

$$
\begin{aligned}
\int_{-\infty}^{\infty}\left[\zeta_{m}^{\sigma}(y) \phi_{n}^{\mu}(y)+\zeta_{n}^{\mu}(y) \phi_{m}^{\mathrm{\alpha}}(y)\right] d y \\
\quad=-2^{1 / 2}\left\{(n+1)\left[(\sigma+\mu) \lambda_{n}-y_{c}\right]+y_{c}\left(\sigma \lambda_{n}-\lambda_{-1}\right)\left(\mu \lambda_{n}-\lambda_{-1}\right)\right\} \delta_{m n} .
\end{aligned}
$$

Thus, (3.13) reduces to

$$
Q^{(I)}\left(x_{E}\right)=-R_{n} p_{o} \exp \left[-i\left(\lambda_{n}-\gamma\right) x^{\prime}\right] \phi_{n}^{+}\left(y^{\prime}\right),
$$

where

$$
R_{n}=\frac{(n+1)\left(\lambda_{n}-\alpha_{-2}\right)+\alpha_{-2}\left(\lambda_{n}-\lambda_{-1}\right)^{2}}{\lambda_{n}\left(\lambda_{n}-\lambda_{-1}\right)\left(\lambda_{n}-\lambda_{-2}\right)}
$$

an expression which is independent of $x_{E}$.

$C A S E(b)$. Here $c_{g}>0$, so that $Q^{(t)}\left(x_{E}\right) \rightarrow 0$ when $x_{E} \rightarrow+\infty$ and we have to compute $Q^{(I)}\left(x_{w}\right)$ for $x_{W}$ sufficiently large and negative. If $x_{W}<x^{\prime},(2.12)$ and (2.13) yield

$$
\begin{aligned}
Q^{(I)}\left(x_{W}\right)= & 2^{-1 / 2} p_{o} \exp \left(-i \alpha_{-2} x_{W}\right) \int_{-\infty}^{\infty} \psi_{o}(y / \sqrt{2})\left\{-\exp \left[-i \alpha_{-2}\left(x^{\prime}-x_{W}\right)\right]\right. \\
& \times \psi_{o}\left(y^{\prime} / \sqrt{2}\right) \psi_{o}(y / \sqrt{2})-\exp \left[-i \alpha_{-1}\left(x^{\prime}-x_{W}\right)\right] \psi_{1}\left(y^{\prime} / \sqrt{2}\right) \psi_{1}(y / \sqrt{2}) \\
& \left.+\gamma \sum_{m=0}^{\infty} \frac{\exp \left[i\left(\lambda_{m}+\gamma\right)\left(x^{\prime}-x_{W}\right)\right]}{\lambda_{m}\left(\lambda_{m}+\lambda_{-1}\right)\left(\lambda_{m}+\lambda_{-2}\right)} \phi_{m}^{-}\left(y^{\prime}\right)\left[\phi_{m}^{-}(y) / 2+\zeta_{m}^{-}(y)\right]\right\} d y .
\end{aligned}
$$


Now, since

$$
\frac{1}{2} \phi_{m}^{\sigma}(y)+\zeta_{m}^{\sigma}(y)=(m+1)^{1 / 2} y \psi_{m+1}(y / \sqrt{2})-(m+1) \psi_{m}(y / \sqrt{2}),
$$

one easily verifies using (3.9) and (3.10) that the integral of each term under the summation sign is equal to zero. The integral of the term associated with the Yanai wave also vanishes, by (3.9); (3.17) then reduces to

$$
Q^{(\prime)}\left(x_{w}\right)=-p_{o} \exp \left(-i \alpha_{-2} x^{\prime}\right) \psi_{o}\left(y^{\prime} / \sqrt{2}\right)
$$

which is again independent of the arbitrary longitude $x_{w}$.

Having computed the integrals $Q^{(t)}(x)$, we can now state our boundary integral formulation of the scattering problem as

$$
\epsilon p\left(\mathbf{r}^{\prime}\right)=\sum_{j} \text { P.V. } \oint_{C_{j}} p(\mathbf{r}) \mathbf{K}\left(\mathbf{r}^{\prime} ; \mathbf{r}\right) \cdot \hat{\mathbf{n}}(s) d s+\eta p^{(I)}\left(\mathbf{r}^{\prime}\right),
$$

where $\epsilon=1$ for $\mathbf{r}^{\prime}$ in the ocean region, $\epsilon=1 / 2$ for $\mathbf{r}^{\prime}$ on any of the contours $C_{1}$, $C_{2}, \ldots, \eta=-R_{n}$ in case (a) and $\eta=1$ in case (b). (For simplicity we have assumed slip boundary conditions at the island boundaries.) The corresponding expression for the velocity vector at a point $\mathbf{r}^{\prime}$ in the ocean region is, by (2.9),

$$
\mathbf{u}\left(\mathbf{r}^{\prime}\right)=-2\left(\rho_{o} c\right)^{-1} \sum_{j} \oint_{c_{j}} p(\mathbf{r}) \mathscr{D}\left(\mathbf{r}^{\prime} ; \mathbf{r}\right) \cdot \hat{\mathbf{n}}(s) d s+\eta \mathbf{u}^{(l)}\left(\mathbf{r}^{\prime}\right)
$$

where the tensor $\mathscr{D}$ is defined by

$$
\mathscr{D}\left(\mathbf{r}^{\prime} ; \mathbf{r}\right)=\left(y^{\prime 2}-y_{c}^{2}\right)^{-1}\left(i y_{c}-y^{\prime} \hat{\mathbf{z}} \times\right) \nabla^{\prime} \mathbf{K}\left(\mathbf{r}^{\prime} ; \mathbf{r}\right) .
$$

(The properties of this influence function were discussed in detail in Part I.) In (3.21), the principal value sign was omitted, since $\mathbf{r}^{\prime}$ is not on the island boundaries.

It is important to observe that (3.20) and (3.21) remain valid if one of the contours $C_{j}$ is assumed to be a continental margin, since this corresponds to an "island" which is unbounded in the meridional direction, and semi-infinite in the zonal direction.

\section{Numerical methods}

Here we discuss the numerical treatment of (3.20) and (3.21) by the Boundary Element Method (BEM) (Brebbia et al., 1984), following the general procedures described in Part I. Let us assume for simplicity that the boundary consists of a single contour $C$ (an island or a continental margin), so that we can discretize it as a broken line with $N+1$ consecutive vertices $\mathbf{r}_{1}, \ldots, \mathbf{r}_{N+1}$. The normal unit vector at the $k$ th boundary element $\Gamma_{k}=\left\{\mathbf{r}_{k}, \mathbf{r}_{k+1}\right\}$ is given by

$$
\hat{\mathbf{n}}_{k}=d_{k}^{-1}\left(\mathbf{r}_{k+1}-\mathbf{r}_{k}\right) \times \hat{\mathbf{z}}
$$


where

$$
d_{k}=\left|\mathbf{r}_{k+1}-\mathbf{r}_{k}\right|
$$

is the length of the element and we assume that $k$ always increases in the direction of $\hat{\mathbf{s}}_{k}=\hat{\mathbf{z}} \times \hat{\mathbf{n}}_{k}$. To treat a boundary which consists of several curves $C_{1}, C_{2}, \ldots$, we would discretize each curve as we have just described and then number the elements consecutively from 1 to the total number of elements.

In the first step of the BEM, we assume that $\mathbf{r}^{\prime}$ in (3.20) is at the boundary and solve the resulting BIE for the boundary pressure distribution. Making the usual approximation that the pressure is nearly constant within each boundary element (Brebbia et al., 1984), we may write the discrete version of (3.20) as

$$
\frac{1}{2} p_{k}=\sum_{j=1}^{N} T_{k j} p_{j}+\eta p_{k}^{(l)}
$$

where

$$
\begin{gathered}
p_{k}=p\left(\mathbf{r}_{k}^{\prime}\right), \quad p_{k}^{(l)}=p^{(l)}\left(\mathbf{r}_{k}^{\prime}\right), \\
\mathbf{r}_{k}^{\prime}=\frac{1}{2}\left(\mathbf{r}_{k+1}+\mathbf{r}_{k}\right), \\
T_{k j}=\hat{\mathbf{n}}_{j} \cdot \int_{\Gamma_{j}} \mathbf{K}\left(\mathbf{r}_{k}^{\prime} ; \mathbf{r}\right) d s,
\end{gathered}
$$

and $T_{k j}$ should be regarded as a principal value integral when $j=k$. When $j \neq k$, we can compute $T_{k j}$ by some numerical quadrature rule, such as the two-point GaussLegendre rule,

$$
T_{k j} \simeq \frac{1}{2} d_{j} \hat{\mathbf{n}}_{j} \cdot\left[\mathbf{K}\left(\mathbf{r}_{k}^{\prime} ; \mathbf{r}_{j}^{\prime}+h d_{j} \hat{\mathbf{s}}_{j}\right)+\mathbf{K}\left(\mathbf{r}_{k}^{\prime} ; \mathbf{r}_{j}^{\prime}-h d_{j} \hat{\mathbf{s}}_{j}\right)\right]
$$

where $h=0.2886751$. When $j=k$, we can write

$$
T_{k k}=\hat{\mathbf{n}}_{k} \cdot\left\{\mathrm{P} \cdot \mathrm{V} \cdot \int_{\Gamma_{k}} \mathbf{K}^{(S)}\left(\mathbf{r}_{k}^{\prime} ; \mathbf{r}\right) d s+\int_{\Gamma_{k}} \mathbf{K}^{(R)}\left(\mathbf{r}_{k}^{\prime} ; \mathbf{r}\right) d s\right\},
$$

where the "singular part" of $\mathbf{K}$ is (see Part I)

$$
\mathbf{K}^{(S)}\left(\mathbf{r}_{k}^{\prime} ; \mathbf{r}\right)=(\gamma / 2 \pi)\left[-2\left(y_{c}-i y_{k}^{\prime} \hat{\mathbf{z}} \times\right)\left(\mathbf{R}_{k} / R_{k}^{2}\right)+\left(i \hat{\mathbf{x}}-2 \gamma y_{k}^{\prime} \hat{\mathbf{y}}\right) \ln R_{k}\right]
$$

with $\mathbf{R}_{k}=\mathbf{r}_{k}^{\prime}-\mathbf{r}, R_{k}=\left|\mathbf{R}_{k}\right|$, and the "regular part" of $\mathbf{K}$ is

$$
\mathbf{K}^{(R)}=\mathbf{K}-\mathbf{K}^{(S)}=\mathbf{O}(1) .
$$

Performing the principal value integration analytically, and the integration of the 
regular part by two-point Gauss-Legendre quadrature, (4.8) becomes

$$
\begin{aligned}
T_{k k} \simeq(\gamma / 2 \pi) d_{k}\left[\ln \left(d_{k} / 2\right)-1\right]\left(i \hat{\mathbf{x}}-2 \gamma y_{k}^{\prime} \hat{\mathbf{y}}\right) \cdot \hat{\mathbf{n}}_{k} \\
\quad+\frac{1}{2} d_{k} \hat{\mathbf{n}}_{k} \cdot\left[\mathbf{K}^{(R)}\left(\mathbf{r}_{k}^{\prime} ; \mathbf{r}_{k}^{\prime}+h d_{k} \hat{\mathbf{s}}_{k}\right)+\mathbf{K}^{(R)}\left(\mathbf{r}_{k}^{\prime} ; \mathbf{r}_{k}^{\prime}-h d_{k} \hat{\mathbf{s}}_{k}\right)\right] .
\end{aligned}
$$

The values of $\mathbf{K}$ and $\mathbf{K}^{(R)}$ appearing in (4.7) and (4.11) can be obtained from a computer routine for the asymptotic evaluation of the influence functions, as described in detail in Part I.

Having computed the matrix elements $T_{k j}$ and the values of $\eta p_{k}^{(l)}$, it is simple to solve the system of equations (4.3) for the boundary pressures $p_{k}$. Since only the boundary is discretized, $N$ will not be large, so that the simple Gaussian elimination method will be adequate for the solution of (4.3).

The final step of the BEM consists of calculating the interior fields at a set of selected points $\mathbf{r}^{\prime}$ within the fluid region. To this end, we use discrete versions of (3.20) (with $\epsilon=1$ ) and (3.21),

$$
\begin{gathered}
p\left(\mathbf{r}^{\prime}\right)=\sum_{k=1}^{N} p_{k} T_{k}+\eta p^{(I)}\left(\mathbf{r}^{\prime}\right), \\
\mathbf{u}\left(\mathbf{r}^{\prime}\right)=-\left(2 / \rho_{o} c\right) \sum_{k=1}^{N} p_{k} \mathbf{D}_{k}+\eta \mathbf{u}^{(I)}\left(\mathbf{r}^{\prime}\right),
\end{gathered}
$$

where

$$
\begin{aligned}
T_{k} & =\hat{\mathbf{n}}_{k} \cdot \int_{\Gamma_{k}} \mathbf{K}\left(\mathbf{r}^{\prime} ; \mathbf{r}\right) d s, \\
\mathbf{D}_{k} & =\int_{\Gamma_{k}} \mathscr{D}\left(\mathbf{r}^{\prime} ; \mathbf{r}\right) \cdot \hat{\mathbf{n}}_{k} d s .
\end{aligned}
$$

These integrals may be computed by the same quadrature rule employed in (4.7) for the computation of $T_{k j}$, provided that the point $\mathbf{r}^{\prime}$ does not lie within a distance of about one deformation radius from the element $\Gamma_{k}$. In this case, the presence of the singularity in the kernels at $\mathbf{r}=\mathbf{r}^{\prime}$ requires that we split the integrand into singular and regular parts, as in (4.8),

$$
\begin{aligned}
& T_{k}=\hat{\mathbf{n}}_{k} \cdot\left\{\int_{\Gamma_{k}} \mathbf{K}^{(S)}\left(\mathbf{r}^{\prime} ; \mathbf{r}\right) d s+\int_{\Gamma_{k}} \mathbf{K}^{(R)}\left(\mathbf{r}^{\prime} ; \mathbf{r}\right) d s\right\}, \\
& \mathbf{D}_{k}=\int_{\Gamma_{k}} \mathscr{D}^{(S)}\left(\mathbf{r}^{\prime} ; \mathbf{r}\right) \cdot \hat{\mathbf{n}}_{k} d s+\int_{\Gamma_{k}} \mathscr{D}^{(R)}\left(\mathbf{r}^{\prime} ; \mathbf{r}\right) \cdot \hat{\mathbf{n}}_{k} d s,
\end{aligned}
$$

where a complete expression for $\mathscr{D}^{(S)}$ is given in Part I. The integrals of the $\mathrm{O}(1)$ regular parts can be computed using the quadrature rule of (4.7), however close to $\Gamma_{k}$ the point $\mathbf{r}^{\prime}$ may be. To compute the integrals of the singular parts on straight-line boundary elements, the analytic expressions given in the Appendix may be used. 


\section{Numerical results}

The above formulation of scattering problems can be directly applied to the various western, eastern and island boundaries of the tropical oceans. A particularly interesting case would be the scattering of Rossby waves by the leaky western Pacific boundary, using a realistic horizontal geometry. In this paper, however, we will illustrate our BIE techniques by a numerical study of the scattering of equatorial Rossby waves of intermediate frequency (periods between 50 and 90 days) by the western Atlantic boundary. Our interest in this problem arose from recent observations of intraseasonal oscillations in the north equatorial Atlantic.

Current meter data from a mooring at $6 \mathrm{~N}, 28 \mathrm{~W}$ indicate that the seasonal cycle of the North Equatorial Countercurrent (NECC) displays intense fluctuations in both eastward and northward velocity, with periods between one and several months (Richardson and Reverdin, 1987). Furthermore, IES data reported by Garzoli (1987) along $28 \mathrm{~W}$ between the Equator and $9 \mathrm{~N}$ suggest the existence of oscillations in the 50-100 day period range, with maximum amplitudes between 3-9N. Spectral peaks centered near the 90-day period have been found in satellite sea surface temperature data at 5N in the western Atlantic (Vianna and Ferreira-Lima, 1992), and also in coastal sea level data from Fortaleza (Vianna and Domingues, 1992). These oscillations appear to be correlated to positive rainfall anomalies in the northern Northeast Brazil. Large-amplitude 50-day oscillations in northward velocity have been discovered in data from a current meter array moored off French Guiana during 1987-88 (Johns et al., 1990). The oscillations have been interpreted as due to westward propagating off-equatorial Rossby waves of negligible group velocity, with vertical structure given by the first baroclinic mode of the equatorial Atlantic. Even though historical Coastal Zone Color Scanner (CZCS) imagery suggests a link between the oscillations and the seasonal North Brazil Current (NBC) retroflection, the persistence of the oscillations over the whole year suggests the presence of excitation mechanisms which are unrelated to instabilities of western boundary currents. In the case of the Indian Ocean, these instabilities generate oscillations which remain largely confined to the western ocean region (Kindle and Thompson, 1989). In the Atlantic, the standing wave pattern observed in sea level, atmospheric pressure and zonal wind records from the gulf of Guinea (Picaut and Verstraete, 1976) might be linked to the 40-50 day atmospheric oscillations (Madden and Julian, 1972) generated in the tropical Pacific and Indian oceans. Since it is possible that this standing wave pattern radiates westward traveling Rossby waves, it would be interesting to study the interference patterns resulting from the reflection of such waves at the western boundary.

In the present linear theory, no mention is made to non-linear effects (such as strong western boundary currents), which could have a strong effect on the reflection process. In the case of the western Atlantic boundary, the NBC could possibly modify our calculated interference patterns. If we consider the $\mathrm{NBC}$ as a zero potential 
vorticity inertial jet (Csanady, 1985), our boundary condition for the pressure will be unchanged by the inclusion of the non-linear terms, provided that the pressure is replaced by the Bernoulli function. If the NBC is a baroclinic current, instead of an inertial jet, then we cannot state that it will not affect the reflection process, although certain studies (e.g., Chang and Philander, 1990) suggest that the effect would be negligible. This issue is important, but is beyond the scope of the present work.

In the simulations that follow, the vertical eigenvalue $c$ will be taken either as $2.17 \mathrm{~m} \mathrm{~s}^{-1}$ or $1.26 \mathrm{~m} \mathrm{~s}^{-1}$, corresponding respectively to the first and second baroclinic modes of the tropical Atlantic (du Penhoat and Treguier, 1985). The corresponding radii of deformation are $R_{o}=(c / 2 \beta)^{1 / 2}=218$ and $167 \mathrm{~km}$, respectively. Recall from Section 2 that for small friction the number of propagating Rossby modes of period $T$ in a given baroclinic mode is given by

$$
N_{R}=\llbracket 1 / 4\left(\left(T / T_{0}\right)^{2}+\left(T_{o} / T\right)^{2}\right)-1 / 2 \rrbracket,
$$

where $T_{o}=2 \pi(2 / \beta c)^{1 / 2}$. The values of $T_{o}$ for the first and second baroclinic modes are 14.6 and 19.2 days, respectively. In all the computations that follow, we have used a dissipation coefficient $A=10^{-8} \mathrm{~m}^{2} \mathrm{~s}^{-3}$ (McCreary et al., 1984). For this value of $A$, short waves are not heavily damped. Since it is difficult to determine the "best" value for the dissipation coefficient, we have chosen a relatively low value, which allows possible short wave effects to appear more clearly. Of course, we expect that such effects will be less intense if a larger value of $A$ is employed.

The model western boundary was taken as the $200 \mathrm{~m}$ isobath, shown for example in Figure $2 \mathrm{~b}$. In order to verify the convergence of the calculated boundary pressure distributions as the boundary element sizes are decreased, we have compared the results obtained with four different discretizations of the boundary. The first two discretizations employ boundary elements with a characteristic size of $120 \mathrm{~km}$ (shorter elements are placed on highly curved parts of the boundary); the first one extends between $20 \mathrm{~S}$ and $20 \mathrm{~N}$, while the second extends from $14.5 \mathrm{~S}$ to $14.5 \mathrm{~N}$. In all cases we have investigated, the boundary pressure distributions obtained by solving (4.3) with these two discretizations were essentially identical. The third and fourth discretizations have boundary elements with characteristic sizes of $80 \mathrm{~km}$ and $60 \mathrm{~km}$, respectively, and both extend from $14.5 \mathrm{~S}$ to $14.5 \mathrm{~N}$. As indicated by Figure 1, the boundary pressure distributions seem to be converging to some limit as the size of the boundary elements is decreased. The computations to be described below make use of the third of the above discretizations.

a. 50-day waves. The number of propagating Rossby modes with period $T=50$ days in the first baroclinic mode is $N_{R}=2$, as can be easily computed from (5.1). The boundary pressure distribution which results when the incident wave is in the first meridional mode is shown in Figure $2 \mathrm{a}$. There is a broad maximum in pressure amplitude, centered near São Luís, and the àmplitude is very small either north of 


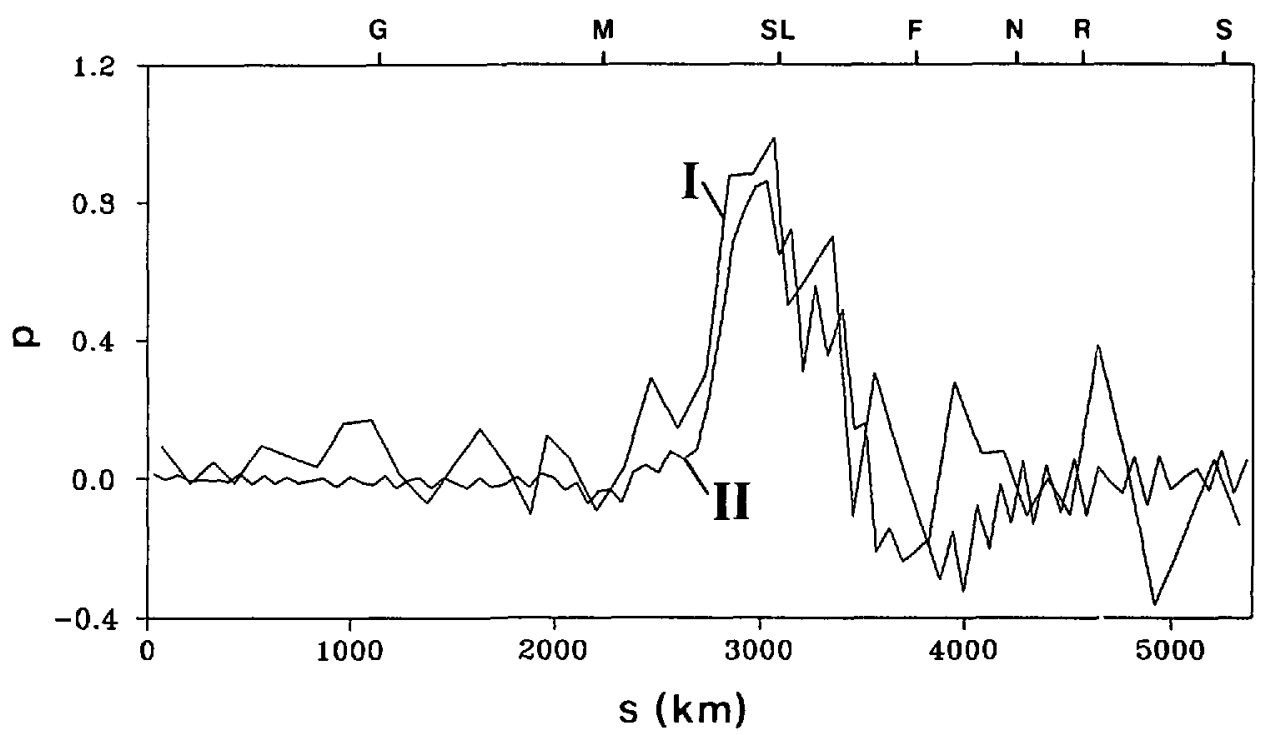

Figure 1. Pressure distribution along the western Atlantic boundary, at a fixed instant, for a second baroclinic mode incident Rossby wave in the first meridional mode with a period of 72 days. Curves I and II show the pressure distributions obtained with boundary discretizations extending from $20 \mathrm{~N}$ to $20 \mathrm{~S}$ and from $14.5 \mathrm{~N}$ to $14.5 \mathrm{~S}$, whose boundary elements have characteristic sizes of 120 and $60 \mathrm{~km}$, respectively. The coordinate $s$ varies from $s=0$ at $14.5 \mathrm{~N}$ to $s=5340 \mathrm{~km}$ at $14.5 \mathrm{~S}$. Letters on the top indicate localities along the coast: Georgetown (G), Maracá Island (M), São Luís (SL), Fortaleza (F), Natal (N), Recife (R) and Salvador (S).

Maracá Island or south of Natal. Between Natal and São Luís, the phase propagates northwestward along the coast, at a speed of $56 \mathrm{~km} /$ day. In the ocean interior, the interference pattern shows a maximum of pressure amplitude between $36-44 \mathrm{~W}$, $2-8 \mathrm{~N}$ (Fig. 2b). The largest amplitude in the field resulting from scattering occurs at $5 \mathrm{~N}, 39 \mathrm{~W}$, and is equal to 3.6 times the maximum amplitude in the incoming wave, which occurs at $4 \mathrm{~N}$. It may be noted from Figure 2 that this maximum occurs at the same longitudes where the largest amplitudes are found along the boundary. Besides, Figure $2 b$ shows that there is considerable amplitude north of the "extreme latitude," or caustic,

$$
y_{e}=2 Q^{1 / 2}=\left[\left(T / T_{o}\right)^{2}+\left(T_{o} / T\right)^{2}\right]^{1 / 2}
$$

(see Part I), which is equal to $6.7 \mathrm{~N}$ in the present case. Since this latitude marks the maximum latitudinal extension of the propagating modes at a given frequency, this suggests that the damped equatorial modes make an important contribution at the amplitude maximum.

The time evolution of the pressure and velocity fields over the region of maximum amplitude is shown at high resolution in Figures 3 and 4 . The zonal wavelength in this 

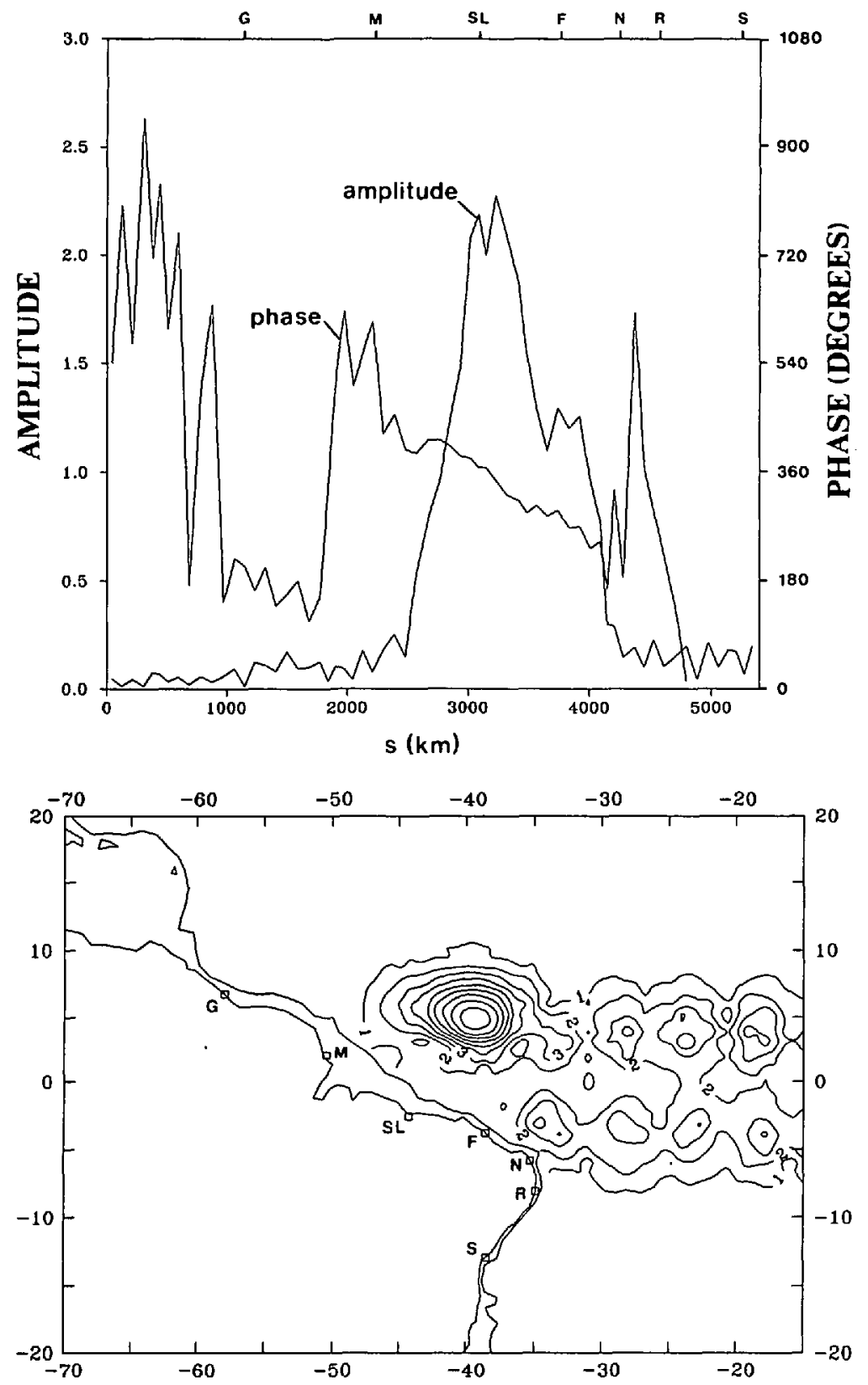

Figure 2. Results for an incident Rossby wave with period $T=50$ days (first baroclinic mode, first meridional mode): (a) boundary solution $p(s)=|p(s)| \exp [-i \varphi(s)]$, where $|p(s)|$ is the amplitude and $\varphi(s)$ is the phase. The phase speed along the boundary is given by $c_{p}=$ $\omega(d \varphi / d s)^{-1}$, so that $d \varphi / d s>0$ corresponds to phase propagation in the direction of increasing $s$. (b) contour map of pressure amplitude, $|p(x, y)|$. The pressure has been scaled in such a way that the maximum amplitude in (b) is 10 . The $s$-scale and the localities along the coast are as in Figure 1. 


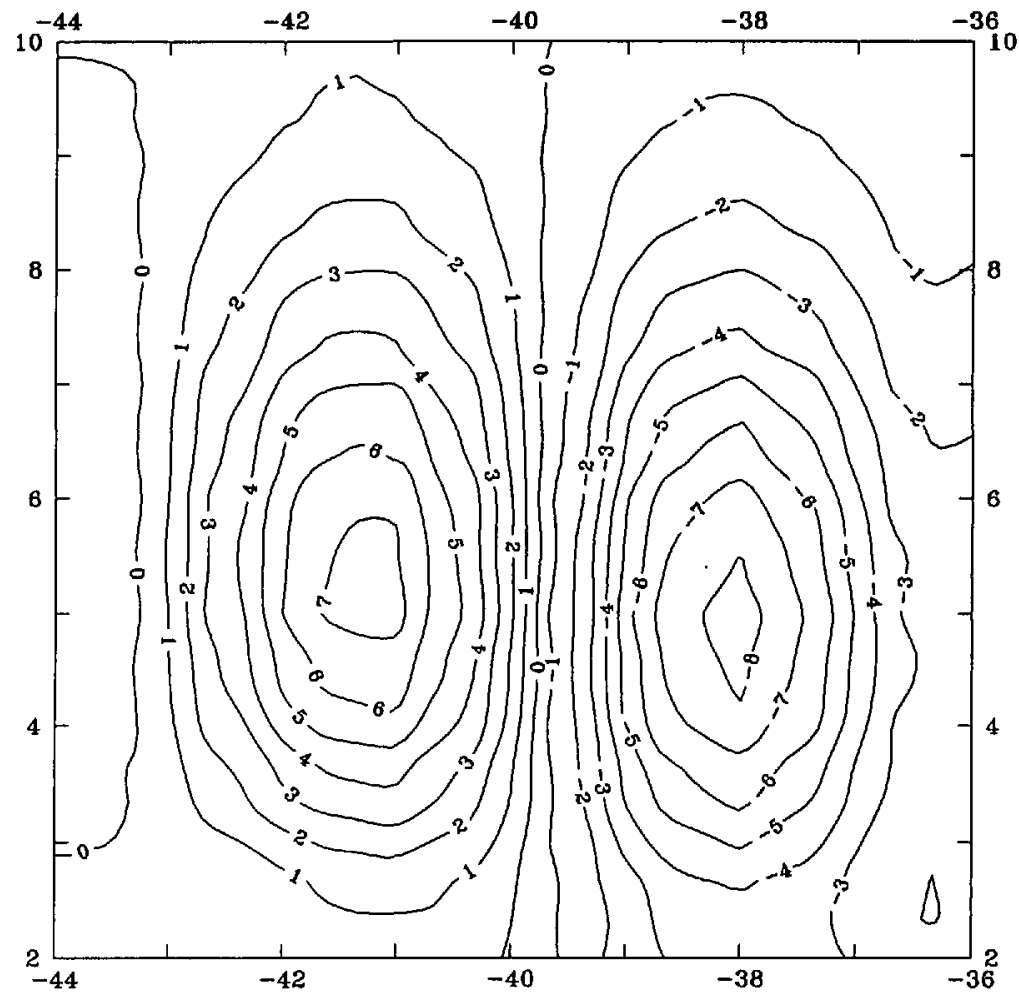

Figure 3. Time evolution of the pressure field over the amplitude maximum of Figure $2 b$ (50-day incident wave, first baroclinic mode, first meridional mode). The figures show two phases which differ by one fourth of the 50 -day cycle. The pressure has been scaled as in Figure 2.

region is approximately $7^{\circ}$ (Fig. 3), while the incident Rossby wave has a wavelength of $23.5^{\circ}$. The real part of the wavenumber of the damped equatorial modes at the 50-day period is given by $-\gamma=-T / 2 T_{o}=-1.72$ in non-dimensional units (see Section 2), which yields a wavelength of $7.1^{\circ}$. This is consistent with our interpretation of the amplitude maximum in terms of damped modes.

The results for an incident wave in the first baroclinic mode and in the second meridional mode are shown in Figure 5. The pressure distribution along the boundary (Fig. 5a) displays a broad relative maximum in amplitude between São Luís and Maracá Island, and a narrower absolute maximum between Fortaleza and Natal. The phase propagation between Natal and Maracá Island is generally northwestward, with two points of stationary phase appearing near São Luís. The phase speed along the coast between Natal and Fortaleza is $54 \mathrm{~km} /$ day to the northwest; between the two points of stationary phase it is $57 \mathrm{~km} /$ day to the southwest; and between São Luís and Maracá Island it is $39 \mathrm{~km} /$ day to the northwest. The amplitude of oscillation in the ocean interior (Fig. 5b) is small west of $38 \mathrm{~W}$. East 


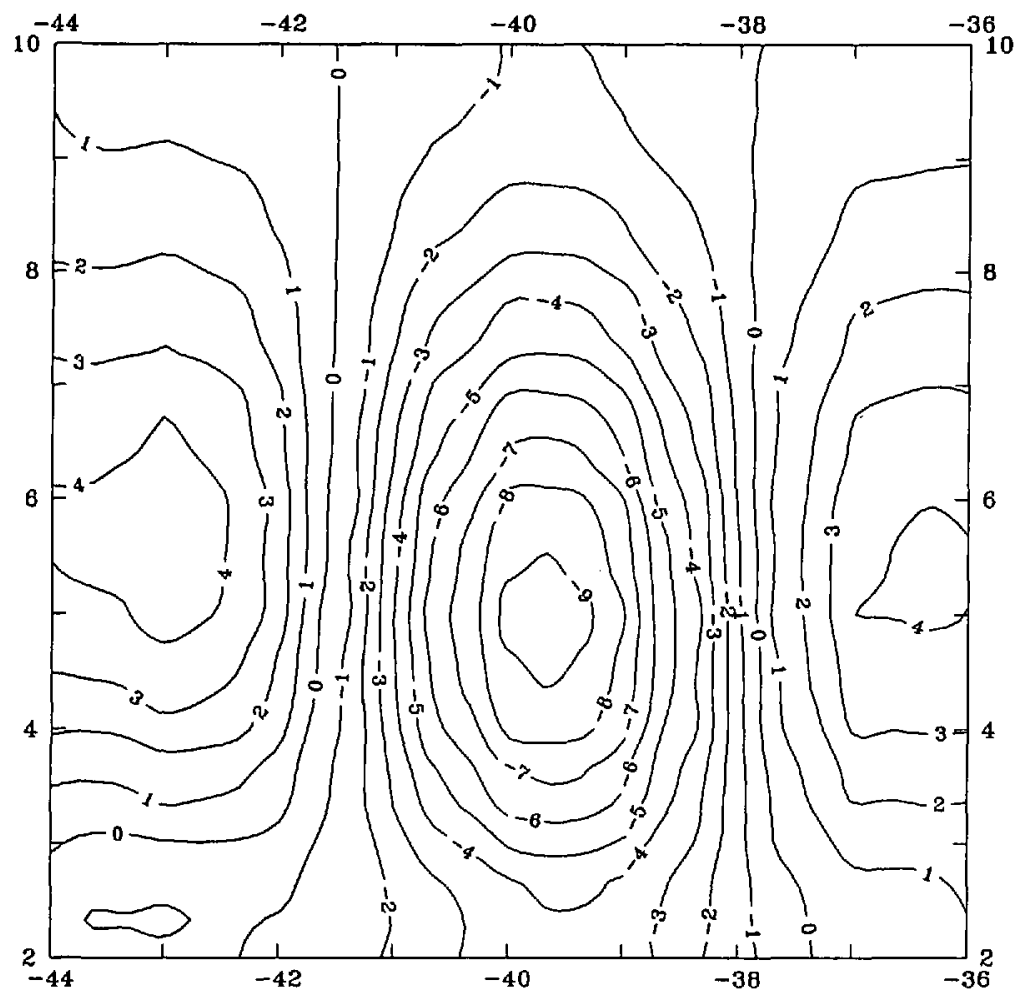

Figure 3. (Continued)

of this meridian, the interference pattern features a sequence of weak amplitude maxima, lying symmetrically with respect to the Equator at $5 \mathrm{~N}$ and $5 \mathrm{~S}$. The maxima have a zonal spacing of about $10^{\circ}$. The amplitude is small poleward of the extreme latitudes $\pm y_{\mathrm{e}}= \pm 6.7^{\circ}$, what suggests that the maxima result from the interference of the equatorial modes with real wavenumbers.

In the second baroclinic mode, (5.1) with $T=50$ days yields $N_{R}=1$. The pressure distribution along the coast induced by a first meridional mode incident wave is qualitatively similar to the one shown in Figure $2 a$. The interference pattern shows a sequence of weak amplitude maxima, similar to those in Figure $5 \mathrm{~b}$.

b. 72-day and 90-day waves (second baroclinic mode). When the incident wave is the first meridional mode Rossby wave, a sharp amplitude maximum appears close to the western boundary, north of the Equator. At the 90-day period (Fig. 6a), the largest amplitude in the field resulting from scattering occurs at $5 \mathrm{~N}, 45 \mathrm{~W}$, and is equal to 4.8 times the maximum amplitude in the incident wave, which occurs at $3 \mathrm{~N}$. The interference pattern for a 72-day incident wave is very similar to Figure 6a, with the difference that the center of the amplitude maximum $(4 \mathrm{~N}, 43 \mathrm{~W})$ is slightly shifted to 


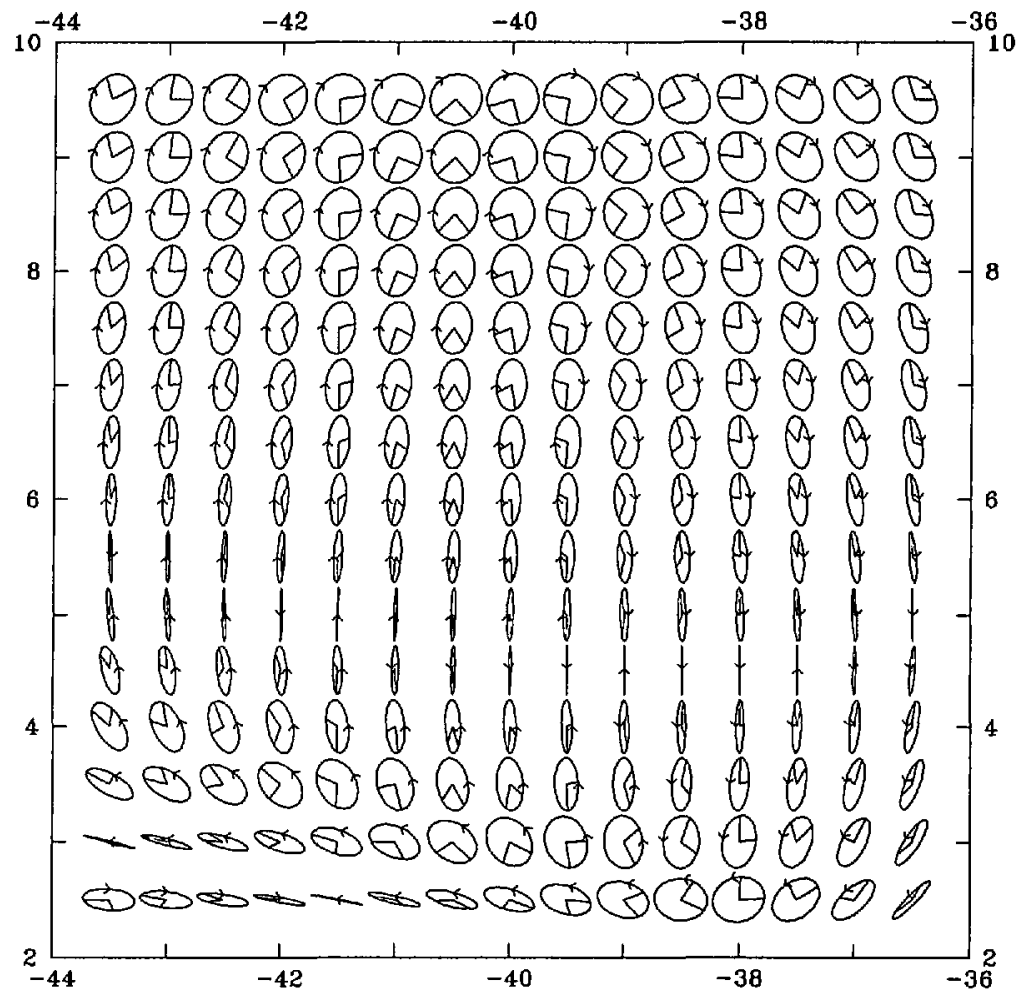

Figure 4. Velocity field over the same region displayed in Figure 3 (50-day incident wave, first baroclinic mode, first meridional mode). In (a), the arrow in each current ellipse gives the sense of rotation of the velocity vector. The pair of straight lines drawn from the center of each ellipse represents the velocity vectors at the phases $\omega t=0$ and $\omega t=90^{\circ}$. For the sake of clarity, the ellipses have been scaled (keeping their shape unchanged) so that their major axes are all of the same size. The actual sizes of the major axes (in arbitrary units) are shown by the contour map in (b).

the southeast. As discussed above, the amplitude maximum is absent when the incident wave has a 50-day period, but we have verified that it appears for waves with periods larger than 60 days. As was the case in Figure $2 b$, the presence of large amplitudes north of the extreme latitude $y_{e}$ (which is $5.7 \mathrm{~N}$ at the 72 -day period and $7.1 \mathrm{~N}$ at the 90-day period) suggests the presence of damped equatorial modes at the amplitude maximum.

When the incident wave is the second meridional mode Rossby wave, the interference pattern has a sharp maximum north of the Equator, which lies more to the east than the maximum induced by a first meridional mode incident wave with the same period (Fig. 6b). As the period is increased, this maximum shifts considerably to the northwest. A second maximum, about $3^{\circ}$ in width, appears close to Natal at the 72-day period; it is directly related to a sharp coastal maximum at that position, 


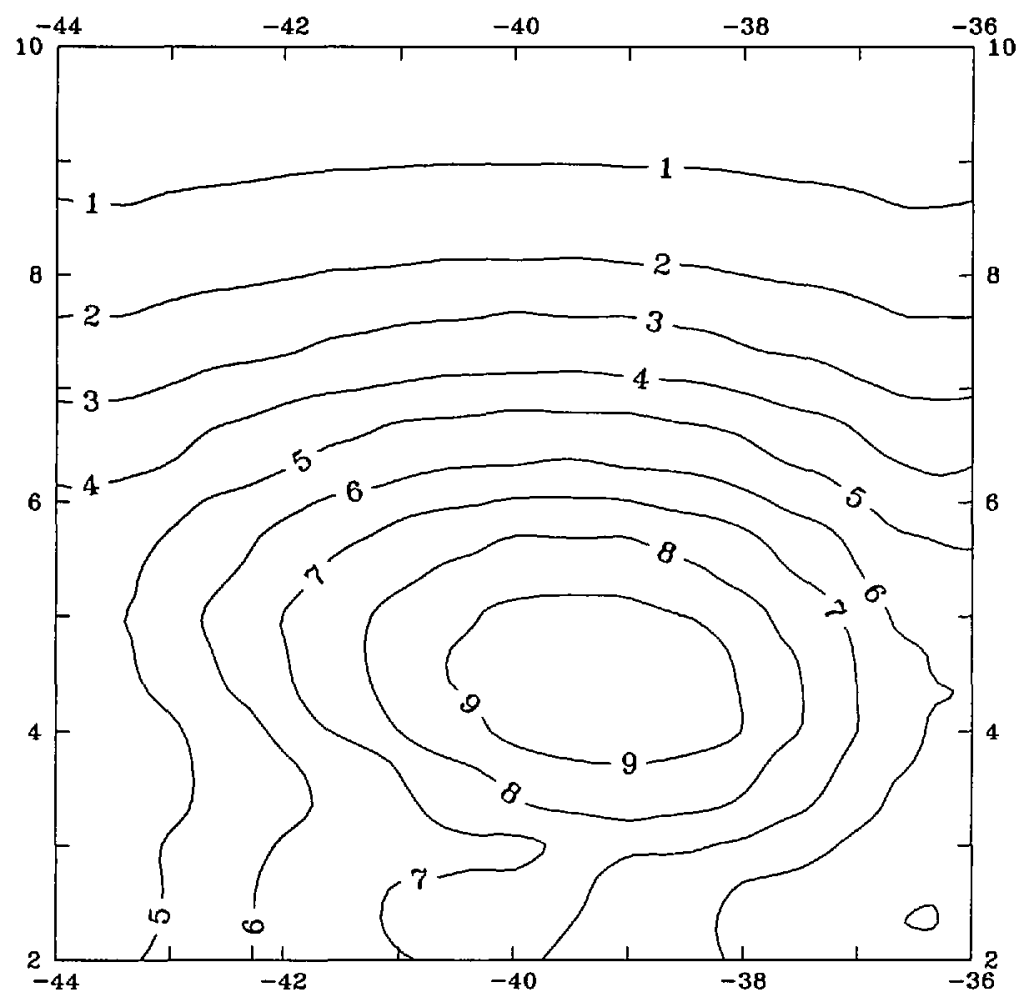

Figure 4. (Continued)

similar to the narrow maximum in Figure 5a. Both maxima exhibit large amplitudes poleward of the extreme latitudes $\pm y_{e}$, so that damped equatorial modes seem to be important in those regions.

c. The numerical results and the observed oscillations. The main purpose of the preceding numerical experiments has been to determine what kinds of interference patterns are produced on a basin-wide scale by the scattering of free Rossby waves from the western Atlantic boundary. The most prominent features in several of our simulations are the amplitude maxima appearing close to western boundary, north of the Equator. Observations of intraseasonal oscillations in the regions of the calculated amplitude maxima are still very few in number, making it difficult to assess whether or not they actually exist. The answer to this question must await for new data and for more analysis of data already available.

Even though the horizontal geometry of our model is realistic, the only process it describes is the (linear) scattering of single-mode free equatorial waves. Thus, with this model we cannot expect to explain in detail the available observations of intraseasonal oscillations, taken at widely separated points in the ocean. What can be done here is to look for features of the observed oscillations which are shared by the 

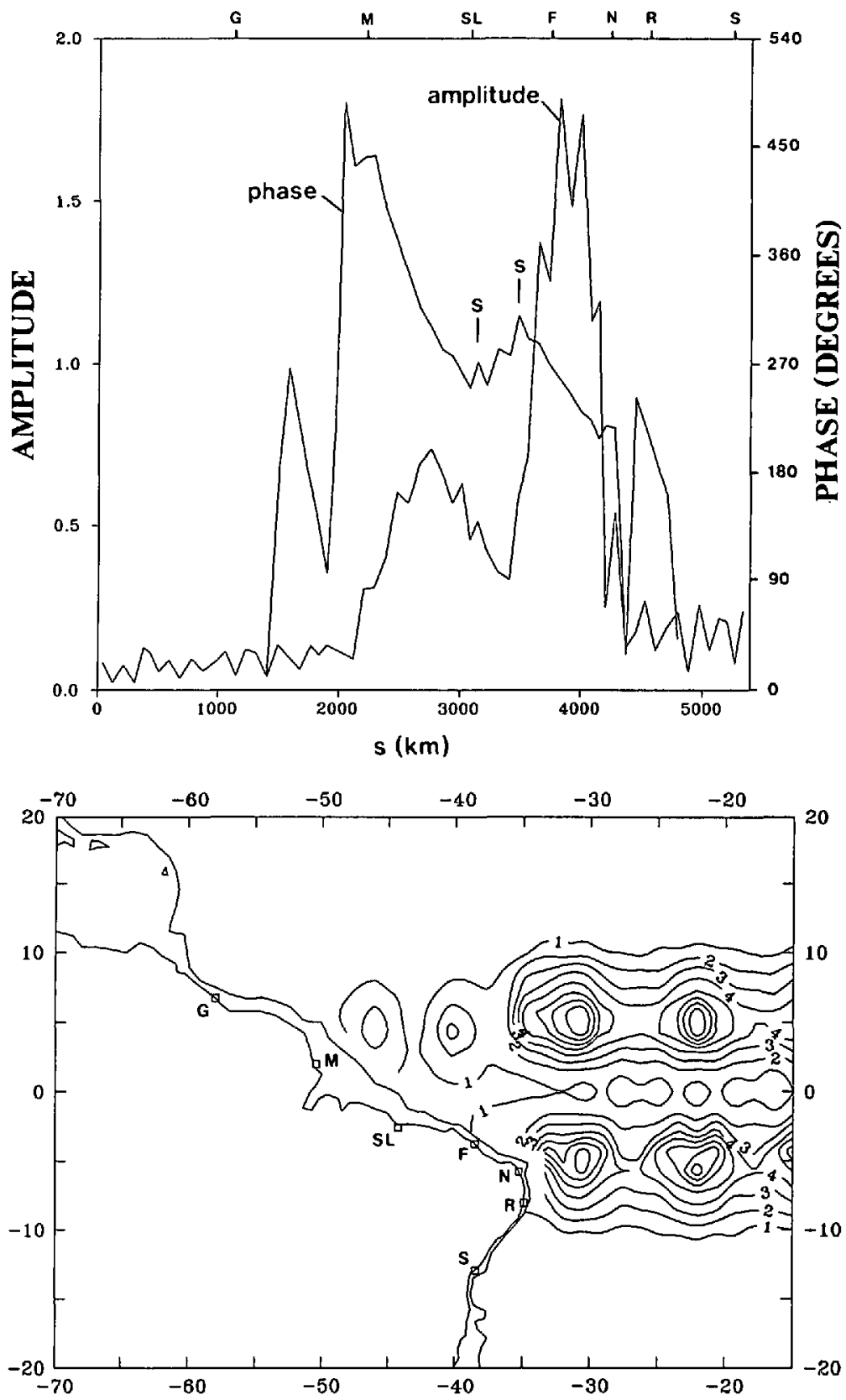

Figure 5. Results for an incident Rossby wave with period $T=50$ days (first baroclinic mode, second meridional mode), displayed as in Figure 2. The stationary phase points mentioned in the text are marked " $S$ " on the phase curve in (a). 

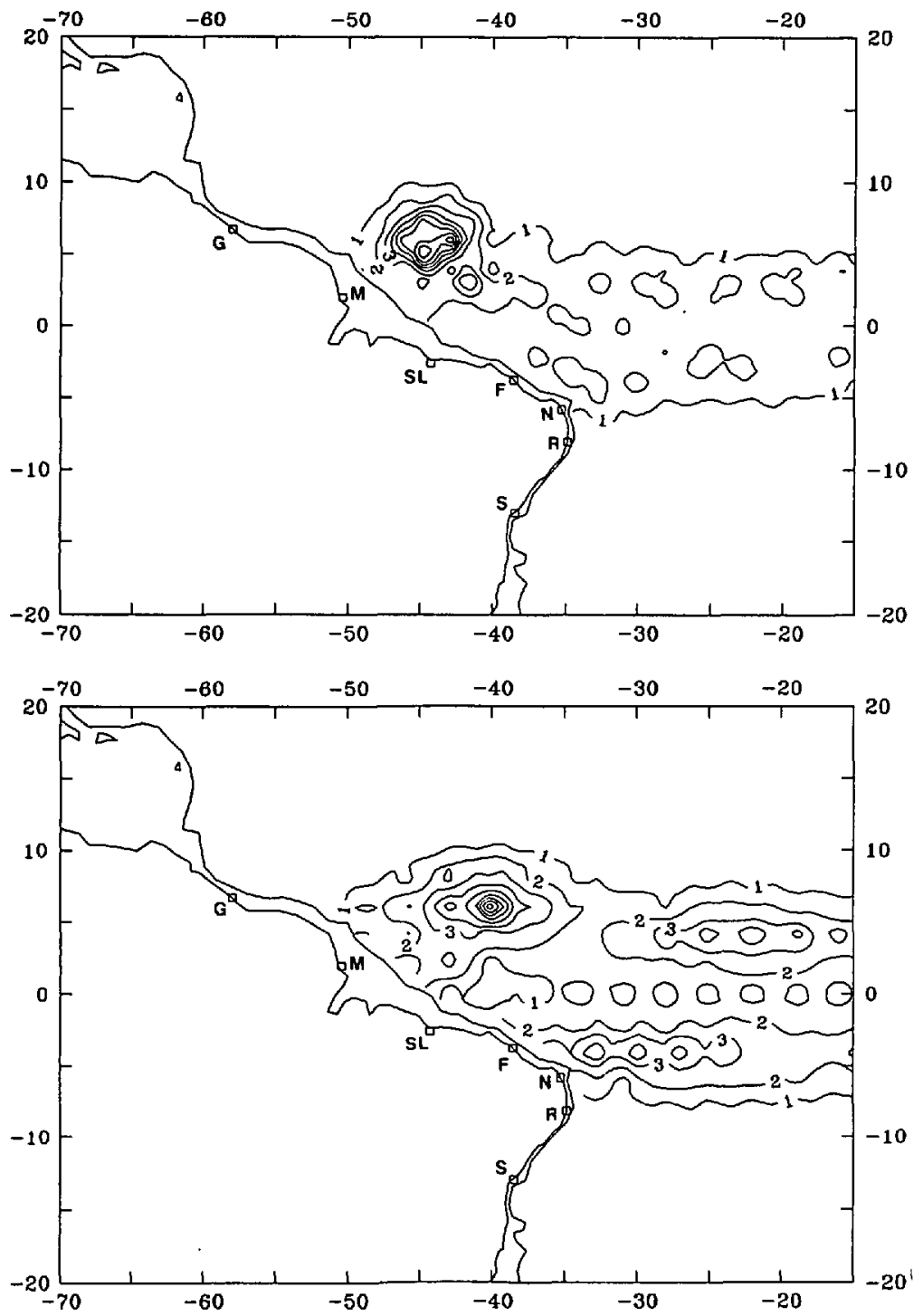

Figure 6. Contour maps of pressure amplitude, $|p(x, y)|$, for incident Rossby waves in the second baroclinic mode: (a) first meridional mode, $T=90$ days; (b) second meridional mode, $T=90$ days. The pressures have been scaled in such a way that the maximum amplitudes in (a) and (b) are equal to 10 (Of course, the scaling factor is different for each map). The abbreviated names of the localities along the coast are defined in the caption of Figure 1.

calculated response due to an incoming free wave of the same period. We consider below three of the observations mentioned at the beginning of this section:

(1) 40-day oscillations at $6 \mathrm{~N}, 28 \mathrm{~W}$. Current meter data from $6 \mathrm{~N}, 28 \mathrm{~W}$ (Richardson and Reverdin, 1987) reveal the existence of 40-day oscillations in the eastern portion 
of the NECC, wherein the current vector rotates clockwise, with the eastward ( $u$; and northward $(v)$ velocity components being approximately $90^{\circ}$ out of phase. At this position, the current vector $\mathbf{u}$ which results from the scattering of the 40-day Rossby wave (first baroclinic mode, first meridional mode) rotates clockwise, and the phase difference between the velocity components is $118^{\circ}$. The current vector $\mathbf{u}^{(l)}$ of the incoming wave at $6 \mathrm{~N}$ also rotates clockwise, and the phase difference between the velocity components is $89^{\circ} 50^{\prime}$. The observed amplitudes of oscillation in $u$ and $v$ are of the same order of magnitude, and the same is true for $\mathbf{u}^{(i)}$. On the other hand, the vector $\mathbf{u}$ has its $u$-and $v$-amplitudes of oscillation in the ratio 1:8. This is due to the excitation of short Rossby waves (with large meridional velocities) at the western boundary. Thus, the incoming wave current vector $\mathbf{u}^{(\prime)}$ resembles the observations more closely than the current vector resulting from scattering. An increase in the dissipation coefficient $A$ would reduce the difference between $\mathbf{u}$ and $\mathbf{u}^{(l)}$, by decreasing the amplitudes of the short Rossby waves.

(2) 73-day oscillations at $3 N, 38 \mathrm{~W}$. A spectral peak in a period band centered at $T=73$ days was noted in IES data from 3N, 38W (Garzoli, 1987). There is a suggestive coincidence between this observation and the sharp amplitude maximum $(2-7 \mathrm{~N}, 35-39 \mathrm{~W})$ which results from the scattering of a 72 -day Rossby wave (second baroclinic mode, second meridional mode). The interference pattern is qualitatively similar to Figure $6 \mathrm{~b}$.

(3) 50-day oscillations at $8 \mathrm{~N}, 52 \mathrm{~W}$. These oscillations were detected with two current meter moorings off French Guiana, one at $7.56 \mathrm{~N}, 52.74 \mathrm{~W}$ (site B) and the other at $8.50 \mathrm{~N}, 52.15 \mathrm{~W}$ (site C) (Johns et al., 1990). The current vectors at the two sites rotate in opposite directions, and the meridional velocities at site $\mathrm{B}\left(v_{B}\right)$ lag those at site $\mathrm{C}\left(v_{C}\right)$ by about $45^{\circ}$. At each site, the current vector rotates in one direction during one half cycle, and in the opposite direction during the other half of the cycle.

We have calculated the current vectors at sites $B$ and $C$ for incident waves in the first baroclinic mode and periods of 45, 50 and 55 days. For $T=45$ days, we have $N_{R}=1$, and for $T=50$ and 55 days, $N_{R}=2$. The calculated vectors exhibit counter-rotation for $T=45$ and 50 days, when the incident wave is in the first meridional mode (Fig. 7). Both the current vectors resulting from the scattering of second meridional mode waves and those of the incident waves under consideration do not exhibit the counter-rotation. Johns et al. (1990) have proposed an interpretation for the oscillation in terms of a local mid-latitude Rossby wave with nearly zonal wave vector, neglecting the interaction between the wave and the ocean boundary. In such a model there is no reason to expect the observed counter-rotation. In our calculation, this effect appears in the 45-50 day period range as a result of interaction between an incoming wave and the boundary geometry; it is not displayed in the incident waves. At $T=45$ days, the major axis of the current ellipse at site $C$ is tilted by nearly $60^{\circ}$ with respect to the zonal direction (Fig. 7). This resembles somewhat the 


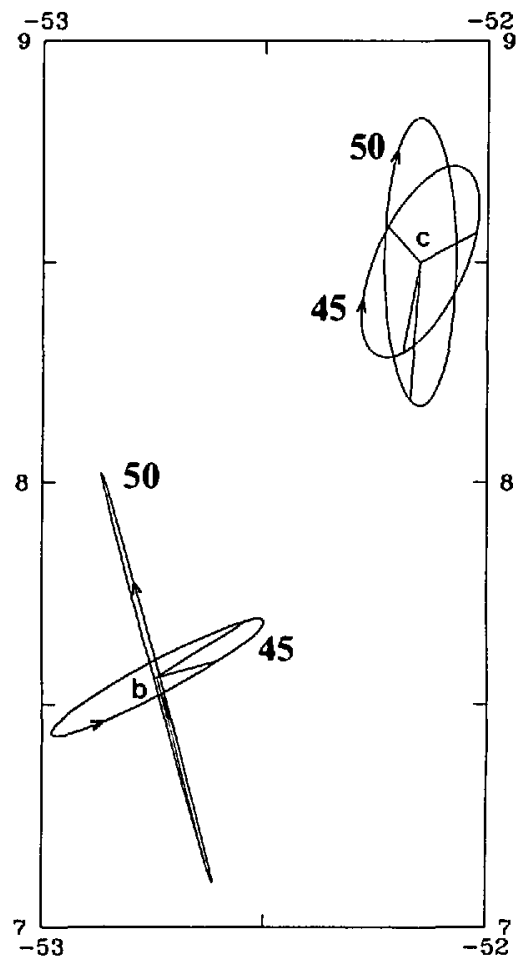

Figure 7. Calculated current vectors at sites B and C of Johns et al. (1990), resulting from the scattering of Rossby waves with periods $T=45$ and 50 days (first baroclinic mode, first meridional mode). The display of the current ellipses is similar to Figure 4, but their sizes are shown to scale.

tilt of the longest current vectors at $100 \mathrm{~m}$ depth at site $\mathrm{C}$, displayed in Figure $3 \mathrm{~b}$ of Johns et al. (1990). The calculated phase lags between the two sites are strongly sensitive to wave period. At $T=50$ days, $v_{C}$ leads $v_{B}$ by $36^{\circ}$, which is reasonably in accord with the observed lag of $45^{\circ}$, but at $T=45$ days the phase difference increases to $135^{\circ}$.

Both our model solution and the mid-latitude Rossby wave proposed by Johns $e t$ al. (1990) fail to exhibit the reversal in the direction of rotation of the currents during half of the cycle. Furthermore, the amplitudes resulting from scattering of equatorial Rossby waves are small west of $50 \mathrm{~W}$ (Fig. $2 \mathrm{~b}$ and $5 \mathrm{~b}$ ). Thus, a single incident plane Rossby wave seems unable to generate certain aspects of the observed 50-day oscillation off French Guiana. A better simulation might possibly be obtained by including the effects of local and remote wind forcing at more than one period.

\section{Discussion}

In this paper we have applied the general boundary integral equation method proposed in Part I to treat the scattering of equatorial waves by islands and 
continental margins of arbitrary geometry. The resulting formulation (Eqs. 3.20 and 3.21 ) is exact in the context of linear theory, and unifies the description of high, intermediate and low frequency dynamics, as well as the treatment of wave scattering by eastern and western ocean boundaries.

From the computational standpoint, the boundary element method is suitable for use with small computers (all the numerical results of this paper were obtained with a personal computer). In fact, little information is needed to describe the geometry of a particular scattering problem, and this can be very easily changed if necessary to increase the boundary resolution. Besides, the number $N$ of boundary elements will normally be $\mathrm{O}\left(10^{2}\right)$, so that the solution of the non-sparse system of equations (4.3) for the boundary pressures can be performed in a short time and with modest memory requirements.

The scattering of intermediate frequency equatorial Rossby waves by a western Atlantic boundary of realistic horizontal geometry was numerically studied using the boundary element method. In all considered wave modes (first and second baroclinic modes, first and second meridional modes), and for low enough frequency, the field resulting from scattering is dominated by an amplitude maximum near the western boundary, north of the Equator. The amplitude at this maximum may be as high as 5 times the maximum amplitude in the incoming wave. The amplitude maxima always appear at the longitude range where maximum amplitudes occur at the coast. Furthermore, the local wavelength at the maxima and the appearance therein of high amplitudes poleward of the extreme latitudes (caustics) $\pm y_{e}$ is consistent with an interpretation in terms of constructive interference of damped equatorial modes (evanescent waves). These modes, excited at the boundary south of the Equator by the incident Rossby wave, can travel with little damping in the meridional direction, since they are affected by damping in the zonal direction only. In this way, the evanescent waves can reach the northern caustic at the latitude $y_{e}$, making there a significant contribution to the pressure field. Therefore, the appearance of the maxima is an effect of linear theory which would probably be missed in a long-wave calculation.

The presence of the maxima is clearly dependent on the slanted boundary geometry, since with a meridional boundary the interference pattern would be symmetrical with respect to the Equator. An amplitude maximum resembling the one found in our study was found in the recent simulation of Moore and McCreary (1990), where a model western Indian Ocean was forced by a zonal wind stress with a period of 60 days. The maximum appears close to the western boundary between $3 \mathrm{~S}$ and $8 \mathrm{~S}$; from our study, we would expect the maximum to lie in the southern hemisphere due to the slant of the African coast from southwest to northeast, as opposed to the slant of the South American coast from southeast to northwest, which produces maxima in the northern hemisphere. 
There is still a lack of observational data on intraseasonal oscillations in the regions where our calculations predict the appearance of amplitude maxima. In this respect, a possible evidence is provided by a spectral peak at $T=73$ days, detected in IES data from 3N, 38W (Garzoli, 1987); large amplitudes are expected at this point from the scattering of the second meridional mode Rossby wave in the second baroclinic mode. On a basin-wide scale, the calculated interference patterns for 50 -day waves (Figs. $2 \mathrm{~b}$ and $5 \mathrm{~b}$ ) seem generally consistent with the observations of 50-70 day oscillations found in IES data from $28 \mathrm{~W}$, those maximum amplitude occurs between $3-9 \mathrm{~N}$, and from 10-34W along the Equator, where the amplitude is low (Garzoli, 1987).

The 40-day current oscillations in the eastern portion of the NECC, observed at $6 \mathrm{~N}, 28 \mathrm{~W}$ (Richardson and Reverdin, 1987) exhibit some similarity to the current induced there by a 40-day free Rossby wave (first baroclinic mode, first meridional mode). The inclusion of the waves reflected at the western boundary sharply increases the amplitude of oscillation of the northward velocity component, but this effect would be weaker if the dissipation coefficient were larger than the one employed in our calculations.

Current oscillations with periods near 50 days have been detected with two current meters moored off French Guiana (Johns et al., 1990). Even though the amplitudes of the fields resulting from the scattering of 50-day waves are small west of $50 \mathrm{~W}$, we have compared the calculated current vectors at both current meter sites with the observed oscillations. For incident Rossby waves with periods between 45 and 50 days (first meridional mode, first baroclinic mode), the calculated current vectors at the two sites exhibit the observed counter-rotation, as a result of the interaction of the incident wave with the western boundary. The phase lag between the sites is strongly sensitive to the wave period, but at $T=50$ days it is reasonably in accord with the observations. On the other hand, our simple model is unable to reproduce the observed reversal in the direction of rotation of the current vectors during half of the 50-day cycle. A more elaborated model for the oscillation should incorporate the effects of some wind-stress distribution over the ocean; to carry out such a study, it would be convenient to develop a time-dependent version of the BIE techniques of the present series of papers. Furthermore, the presence of the strong North Brazil Current in the region could indeed require the use of a non-linear model.

Although we have not investigated the scattering of waves with periods longer than 90 days, our results suggest that in these cases large amplitudes are also to be found north of the Equator, near the western Atlantic boundary. This seems consistent with the presence of a strong maximum in the annually averaged eddy kinetic energy, centered at $5 \mathrm{~N}, 47 \mathrm{~W}$, as derived from historical ship drift data (Richardson and Philander, 1987). Furthermore, the present calculations suggest that scattering effects may be important in the interpretation of recent altimetric observations of sea 
level in the equatorial Atlantic from Geosat (Carton, 1989), GEOS3 and Seasat (Menard, 1988), which exhibit high-amplitude features north of the Equator and west of $35 \mathrm{~W}$.

Acknowledgments. We are grateful to Admiral Fernando Freitas and Admiral Maximiano da Fonseca from the Diretoria de Transportes and to Dr. Milton Francke, superintendent of DEPEX, all from PETROBRÁS, for their encouragement and for the support that made this research possible. One of us (P.R.H.) has been supported by a PETROBRÁS fellowship, under grant SEDES-30239/89. P.R.H. wishes also to thank Angelo Shimabuko for the use of his personal computer in the numerical simulations of this paper.

\section{APPENDIX}

\section{Evaluation of near-singular integrals}

The singular parts of the kernels are linear combinations of homogeneous functions of $\mathbf{R}=\mathbf{r}^{\prime}-\mathbf{r}$ and of logarithmic functions of $R=|\mathbf{R}|$ (see Part I). Consider a straight-line boundary element $E=\left\{\mathbf{r}_{1}, \mathbf{r}_{2}\right\}$, and a point $\mathbf{r}^{\prime}$ not on the element. The length element along $E$ is

$$
d s=\left|\mathbf{r}_{2}-\mathbf{r}_{1}\right| d \xi=d_{12} d \xi
$$

where $0 \leq \xi \leq 1$, the normal vector to $E$ is

$$
\hat{\mathbf{n}}=d_{12}^{-1}\left(\mathbf{r}_{2}-\mathbf{r}_{1}\right) \times \hat{\mathbf{z}}
$$

and for $\mathbf{r}$ on $E$ we have

$$
\begin{aligned}
\mathbf{R} & =\left(\mathbf{r}^{\prime}-\mathbf{r}_{1}\right)+\xi\left(\mathbf{r}_{1}-\mathbf{r}_{2}\right), \\
R^{2} & =A \xi^{2}+B \xi+C
\end{aligned}
$$

with

$$
A=d_{12}^{2}, \quad B=2\left(\mathbf{r}_{1}-\mathbf{r}_{2}\right) \cdot\left(\mathbf{r}^{\prime}-\mathbf{r}_{1}\right), \quad C=\left|\mathbf{r}^{\prime}-\mathbf{r}_{1}\right|^{2} .
$$

Thus, from (A.3) and (4.9) we get

$$
\begin{gathered}
\hat{\mathbf{n}} \cdot \int_{E} \mathbf{K}^{(s)}\left(\mathbf{r}^{\prime} ; \mathbf{r}\right) d s=(\gamma / \pi)\left\{\left[y_{c}\left(x_{01} y_{12}-x_{12} y_{01}\right)\right.\right. \\
\left.\left.+i y^{\prime}\left(x_{01} x_{12}+y_{01} y_{12}\right)\right] I_{1}+i y^{\prime} d_{12}^{2} I_{2}-1 / 4\left[2 \gamma x_{12} y^{\prime}+i y_{12}\right] I_{3}\right\}
\end{gathered}
$$

where

$$
\begin{gathered}
x_{01}=x^{\prime}-x_{1}, \quad y_{01}=y^{\prime}-y_{1}, \quad x_{12}=x_{1}-x_{2}, \quad y_{12}=y_{1}-y_{2}, \\
I_{1}=\int_{0}^{1} \frac{d \xi}{R^{2}}, \quad I_{2}=\int_{0}^{1} \frac{\xi d \xi}{R^{2}}, \quad I_{3}=\int_{0}^{1} \ln R^{2} d \xi .
\end{gathered}
$$


Using the expression for $\mathscr{D}^{(S)}\left(\mathbf{r}^{\prime} ; \mathbf{r}\right)$ given in Part I, we find analogously

$$
\begin{array}{r}
\hat{\mathbf{x}} \cdot \int_{E} D^{(s)}\left(\mathbf{r}^{\prime} ; \mathbf{r}\right) \cdot \hat{\mathbf{n}} d s=(\gamma / \pi i)\left\{d_{12}^{2}\left(y_{12} I_{4}+2 y_{01} I_{5}\right)\right. \\
+\left[2 x_{12} x_{01} y_{01}+y_{12}\left(y_{01}^{2}-x_{01}^{2}\right)\right] I_{6}+2 i \gamma\left[1 / 2\left(x_{01} y_{12}-x_{12} y_{01}\right)\left[\left(y_{12}^{2}-x_{12}^{2}\right) I_{4}\right.\right. \\
\left.\left.+\left(y_{01}^{2}-x_{01}^{2}\right) I_{6}\right]+\left(x_{01} x_{12}-y_{01} y_{12}\right)\left(x_{12} y_{01}-y_{12} x_{01}\right) I_{5}\right] \\
\left.\quad-1 / 16\left[y_{12}\left(y^{\prime 2}+4 \lambda_{-1} \lambda_{-2}\right)+2 i x_{12} y^{\prime} y_{\mathrm{c}}\right] I_{3}\right\},
\end{array}
$$

$$
\begin{aligned}
& \hat{\mathbf{y}} \cdot \int_{E} \mathscr{D}^{(s)}\left(\mathbf{r}^{\prime} ; \mathbf{r}\right) \cdot \hat{\mathbf{n}} d s=(\gamma / \pi i)\left[-d_{12}^{2}\left(x_{12} I_{4}+2 x_{01} I_{5}\right)\right. \\
&-\left[2 y_{12} x_{01} y_{01}-x_{12}\left(y_{01}^{2}-x_{01}^{2}\right)\right] I_{6}+i \gamma\left[\left(y_{01}^{2}-x_{01}^{2}\right)\left[\left(x_{12} x_{01}+y_{12} y_{01}\right) I_{6}+2 d_{12}^{2} I_{5}\right]\right. \\
&\left.+\left[y_{01} y_{12}\left(y_{12}^{2}+3 x_{12}^{2}\right)-x_{01} x_{12}\left(x_{12}^{2}+3 y_{12}^{2}\right)\right] I_{4}+d_{12}^{2} I_{2}+2 x_{12} x_{01} I_{1}\right] \\
&\left.+1 / 16\left[x_{12}\left(y^{\prime 2}+y_{c}^{2}-3 y_{c}^{-2}\right)-2 i y_{12} y^{\prime} y_{c}\right] I_{3}\right\},
\end{aligned}
$$

where

$$
I_{4}=\int_{0}^{1} \frac{\xi^{2} d \xi}{R^{4}}, \quad I_{5}=\int_{0}^{1} \frac{\xi d \xi}{R^{4}}, \quad I_{6}=\int_{0}^{1} \frac{d \xi}{R^{4}}
$$

In view of (A.3), the integrals $I_{1}, \ldots, I_{6}$ can be expressed as

$$
\begin{aligned}
& I_{1}=2 T, \quad I_{2}=-\frac{1}{A}\left[B T-\frac{1}{2} \ln \frac{S}{C}\right], \\
& I_{3}=\frac{1}{A}\left[T \Delta+\frac{1}{2} B \ln \frac{S}{C}\right]+\ln S-2, \\
& I_{4}=\frac{1}{\Delta}\left[4 C T-\frac{B+2 C}{S}\right], \quad I_{5}=-\frac{1}{\Delta}\left[2 B T-\frac{2 A+B}{S}\right], \\
& I_{6}=\frac{1}{\Delta}\left[4 A T+\frac{A(2 C-B)-B^{2}}{C S}\right],
\end{aligned}
$$

where

$$
\begin{aligned}
& \Delta=4 A C-B^{2}, \quad T=\frac{1}{\sqrt{\Delta}}\left[\tan ^{-1} \frac{(2 A+B)}{\sqrt{\Delta}}-\tan ^{-1} \frac{B}{\sqrt{\Delta}}\right], \\
& S=A+B+C .
\end{aligned}
$$

\section{REFERENCES}

Brebbia, C. A., J. C. F. Telles and L. C. Wrobel. 1984. Boundary Element Techniques, Springer-Verlag, Berlin, $463 \mathrm{pp}$.

Cane, M. A. and Y. du Penhoat. 1982. The effect of islands on low-frequency equatorial motions. J. Mar. Res., 40, 937-962. 
Cane, M. A. and P. R. Gent. 1984. Reflection of low-frequency equatorial waves at arbitrary western boundaries. J. Mar. Res., 42, 487-502.

Carton, J. A. 1989. Estimates of sea level in the tropical Atlantic ocean using Geosat altimetry. J. Geophys. Res., 94C, 8029-8039.

Chang, P. and S. G. H. Philander. 1990. Quasi-geostrophic oceanic adjustment in the presence of mean currents. Dyn. Atmos. Oceans, 14, 387-414.

Clarke, A. J. 1991a. On the reflection and transmission of low-frequency energy at the irregular western Pacific Ocean boundary. J. Geophys. Res., 96, (supplement), 3289-3305.

- 1991b. Low frequency reflection from a non-meridional eastern ocean boundary and the use of coastal sea level to monitor eastern Pacific equatorial Kelvin waves. J. Phys. Oceanogr., (submitted).

Csanady, G. T. 1985. A zero potential vorticity model of the North Brazilian Coastal Current. J. Mar. Res., 43, 552-579.

Delcroix, T., G. Eldin and J. Picaut. 1989. GEOSAT sea level anomalies in the western equatorial Pacific during the 1986-87 El Niño, elucidated as equatorial Kelvin and Rossby waves. Proceedings of the Western Pacific International Meeting and Workshop on TOGA COARE, 259-268. Centre ORSTOM de Nouméa, New Caledonia.

du Penhoat, Y. and M. A. Cane. 1989. Effect of low latitude western boundary gaps on the reflection of equatorial motions. Proceedings of the Western Pacific International Meeting and Workshop on TOGA COARE, 335-342. Centre ORSTOM de Nouméa, New Caledonia.

du Penhoat, Y, and A. M. Treguier. 1985. The seasonal linear response of the tropical Atlantic Ocean. J. Phys. Oceanogr., 15, 316-329.

Enfield, D. B. 1989. El Niño, past and present. Reviews of Geophysics, 27, 159-187.

Garzoli, S. L. 1987. Forced oscillations on the equatorial Atlantic basin during the Seasonal Response of the Equatorial Atlantic Program (1983-1984). J. Geophys. Res., 92C, 50895100.

Gill, A. E. 1983. An estimation of sea-level and surface-current anomalies during the $1972 \mathrm{El}$ Niño and consequent thermal effects. J. Phys. Oceanogr., 13, 586-606.

Johns, W. E., T. N. Lee, F. A. Schott, R. J. Zantopp and R. H. Evans. 1990. The North Brazil Current Retroflection: seasonal structure and eddy variability. J. Geophys. Res., 95C, 22103-22120.

Kindle, J. and J. D. Thompson. 1989. The 26- and 50-day oscillations in the western Indian Ocean: Model results. J. Geophys. Res., 94C, 4721-4736.

Luyten, J. R. and D. H. Roemmich. 1982. Equatorial currents at the semi-annual period in the Indian Ocean. J. Phys. Oceanogr., 12, 406-413.

Madden, R. and P. Julian. 1972. Description of global scale circulation cells in the tropics with a 40-50 day period. J. Atm. Sci., 29, 1109-1123.

McCalpin, J. D. 1987. A note on the reflection of low-frequency equatorial Rossby waves from realistic western boundaries. J. Phys. Oceanogr., 17, 1944-1949.

McCreary, J. P. 1981. A linear stratified ocean model of the equatorial undercurrent. Phil. Trans. Roy. Soc. London, Ser. A, 298, 605-635.

McCreary, J. P., J. Picaut and D. W. Moore. 1984. Effects of remote annual forcing in the eastern tropical Atlantic Ocean. J. Mar. Res., 42, 45-81.

Menard, Y. 1988. Observing the seasonal variability in the tropical Atlantic from altimetry. J. Geophys. Res., 93C, 13967-13978.

Moore, D. W. and J. P. McCreary. 1990. Excitation of intermediate-frequency equatorial 
waves at a western ocean boundary: with application to observations from the Indian Ocean. J. Geophys. Res., 95C, 5219-5231.

Picaut, J., B. Camusat, T. Delcroix, M.J. McPhaden and A.J. Busalacchi. 1989. Surface equatorial flow anomalies in the Pacific Ocean during the 1986-87 ENSO using GEOSAT altimeter data. Proceedings of the Western Pacific International Meeting and Workshop on TOGA COARE, 301-309. Centre ORSTOM de Nouméa, New Caledonia.

Picaut, J. and J. Verstraete. 1976. Mise en évidence d'une onde de 40-50 jours de période sur les côtes du golfe de Guinée. Cah. ORSTOM, sér. Océanogr., vol. XIV, 3-14.

Richardson, P. L. and S. G. H. Philander. 1987. The seasonal variations of surface currents in the tropical Atlantic Ocean: A comparison of ship drift data with results from a general circulation model. J. Geophys. Res., 92C, 715-724.

Richardson, P. L. and G. Reverdin. 1987. Seasonal cycle of velocity in the Atlantic north equatorial countercurrent as measured by surface drifters, current meters, and ship drifts. J. Geophys. Res., 92C, 3691-3708.

Rowlands, P. B. 1982. The flow of equatorial Kelvin waves and the Equatorial Undercurrent around islands. J. Mar. Res., 40, 915-936.

Vianna, M. L. and M. Domingues. 1992. Intraseasonal oscillations in sea level and precipitation in Northeast Brazil. (in preparation).

Vianna, M. L. and R. A. Ferreira-Lima. 1992. Characterization of interannual variability of intraseasonal oscillations in the tropical Atlantic through MC SST/AVHRR data. J. Geophys. Res., (submitted).

Vianna, M. L. and P. R. Holvorcem. 1992. Integral equation approach to tropical ocean dynamics, Part I-Theory and computational methods. J. Mar. Res., 50, 1-31.

Weisberg, R. H., J. H. Hickman, T. Y. Tang and T. J. Weingartner. 1987. Velocity and temperature observations during the Seasonal Response of the Equatorial Atlantic Experiment at $0^{\circ}, 28 \mathrm{~W}$. J. Geophys. Res., $92 \mathrm{C}$, 5061-5075.

Weisberg, R. H. and T. Y. Tang. 1985. On the response of the equatorial thermocline in the Atlantic Ocean to the seasonally varying trade winds. J. Geophys. Res., 90C, 7177-7128.

1987. Further studies on the response of the equatorial thermocline in the Atlantic

Ocean to the seasonally varying trade winds. J. Geophys. Res., 92C, 3709-3727.

- 1990. A linear analysis of equatorial Atlantic Ocean thermocline variability. J. Phys. Oceanogr., 20, 1813-1825.

Yoon, J. 1981. Effects of islands on equatorial waves. J. Geophys. Res., 86C, 10913-10920. 